\title{
Assessing Process and Coating Reliability Through Monitoring of Process and Design Relevant Coating Properties
}

\author{
Gopal Dwivedi, Travis Wentz, Sanjay Sampath, and Toshio Nakamura
}

(Submitted August 11, 2009; in revised form October 14, 2009)

\begin{abstract}
Thermal spray in general, plasma spray in particular, is a highly complex process with numerous interacting variables associated with generation of the spray stream, deposit formation dynamics, and the resultant property linkages. Compounding this variability further are both the spatial (different booths and different locations) and temporal (process start-stops, hardware degradation, operator etc.) effects. As such, an understanding of process and coating consistency and variability offers significant challenges. Recent scientific advances as well as measurement tools have enabled elucidation of the intrinsic variabilities associated with each of the process sub-steps; however, integrated understanding of the system level reliability is still lacking. This article seeks an integrated assessment of process and coating reliability through systematic measurements of variabilities during each stage of the process subjected to different operating parameters. Through critical examination of first-order process maps, the influence of process parameters on particle state is reviewed for repeated spray runs with a single parameter effect as well as across a spectrum of process parameters. In addition, influence of these changes on designrelevant coating properties were obtained for plasma-sprayed zirconia through recourse to novel in situ and ex situ substrate curvature measurements. Finally, the implications of such integrated reliability studies have been explored through collaborative experiments conducted in the industrial sites.
\end{abstract}

Keywords elastic properties, plasma spray, process diagnostics, process maps, reliability, thermal barrier coatings, variability, yttria-stabilized zirconia

\section{Background}

Plasma spray is a highly complex process with a multitude of interdependent variables that control the characteristics of the deposited material. The stochastics of the process are significantly associated with the generation of the molten particle spray, for the formation of the deposit through successive impingement of the droplets, which can affect the deposit characteristics and properties in numerous ways (Ref 1-5). Of further importance is the two back-to-back phase changes (melting and solidification) experienced by the material, both of which occurs in very short time scales (in tens of microseconds) and are also subjected to strong temperature gradients (Ref 6,7). Finally, the deposit microstructure itself is highly complex comprising innumerable defects and interfaces of different

Gopal Dwivedi and Sanjay Sampath, Center for Thermal Spray Research, Department of Materials Science and Engineering, State University of New York, Stony Brook, NY; and Travis Wentz and Toshio Nakamura, Center for Thermal Spray Research, Department of Mechanical Engineering, State University of New York, Stony Brook, NY. Contact e-mails: gdwivedi@ic.sunysb.edu and gopal.dvd@gmail.com.

length scales resulting in difficulties in interpreting and quantifying the properties (Ref 8-10). These complexities have resulted in significant challenges in our ability to manipulate and control the plasma spray process to reliably achieve desired properties and performance.

In recent years, much progress has been made both at the scientific level and in diagnostic instrumentation, which has enabled probing the process-structure-property linkages together with concomitant growth in applications. However, issues relative to process and coating reliability continue to confound academic and industrial researchers. This is particularly true for the ceramic thermal barrier coating (TBC) system where new demands in engine performance require coatings to play the role of "prime reliance," necessitating advances in both process engineering as well as in coating life prediction. Prime reliance refers to designed-in performance of the coating system along with a predictable life of operation (Ref 11, 12). Prime reliance is affected both by the intrinsic variability of the deposited material and environmental influence, which determine its durability. The external factors include, but not limited to, oxidation, thermal cycling, erosion, and foreign object damage.

Although much remains to be done to enable prime reliance of present day TBC, producing reliable and reproducible coatings is a necessary first step. Enhancing process reliability will reduce rework, improve process efficiency, materials usage, and provide overall confidence for life prediction by reducing part-to-part coating variability. Achieving this is a non-trivial task considering the number of steps involved in industrial coating generation. 
Although progress has been made in many of the areas associated with plasma spray processing of $\mathrm{TBC}$, they have to a large extent been piece-meal addressing one or more of the attributes and, generally, not considered through an integrated framework. This is particularly true with respect to understanding and defining coating properties and their associated variability. This article is a first step in this endeavor and attempts to integrate process science with material science with specific emphasis of examining reliability. A key attribute is determining design-relevant properties of ceramic TBCs (compliance and thermal conductivity) through a novel curvature measurement technique and utilizes these new strategies to quantify coating reproducibility both in academic and industrial setting.

Before addressing the specific issues with respect to process and coating reliability, it is appropriate to clarify the nuances and terminologies associated with understanding reliability particularly with respect to industrial plasma spray operations of TBCs. Variability in industrial TBC production results from temporal or time-dependent variation within a single spray booth, as well as through spatial variation in TBC fabrication arising from multiple spray booth within a location or locations around the world. The former considers not only intrinsic variabilities within a specified process, but also contemplates extrinsic issues such as hardware degradation, environment, and feedstock. The latter is important to examine variabilities in coating processing due to different booth configurations, spray torches, and even subtle environmental differences (e.g., humidity) at locations around the world. As such, it is worthwhile to clarify the definitions specific to this application. In this article, this is addressed through the following definitions of process repeatability, coating reproducibility, and process/coating reliability.

- Repeatability can be defined as variation in the measurements obtained when one person measures the same attribute with the same measuring equipment, or it could be the variation in measurements obtained when one person takes multiple measurements using the same instrument and techniques on the same parts or items. It can be enhanced by improving the least count of the instrument and setting a standard procedure for the measurements. In the case of plasma spraying of TBCs, process repeatability is a term that captures temporal variations in the process/coating within a single booth/site where the operations are routinely repeated for a given application/specification. Thus, the focus here is the act of repeating a process to achieve the same coating. Hardware degradation such as electrode wear can be considered within the context of achieving process repeatability within a single spray environment.

- Reproducibility is generally defined by the variation in average measurements obtained when two or more people measure the same parts or items using the same measuring technique. In the case of plasmasprayed TBCs, the term reproducibility is more commonly used to define spatial variations, for instance, when two or more booths/sites seek to reproduce the same coating either through the same or similar processes. Note that, here, the goal is the outcome and not the steps associated with achieving the goal unlike the repeatability case.

- Reliability is a system level definition encompassing all the aspects of the process and coating so as to meet the requisite performance goals. Reliability of a coating is the probability that it will adequately perform its specified purpose for a specified period of time under specified environmental conditions. As such, the term reliability captures all the aspects of repeatability and reproducibility and sets fundamental benchmarks at each step of the process.

Each stake holder has a different view of the above three R's (repeatability, reproducibility, and reliability). System producers who specify the coating for the application are driven by the objectives of the application. In the case of TBCs, they seek reliable coating performance which can be achieved by reproducible coatings both in time and spatial domain. On the other hand, process engineers and coating applicators seek a reliable process that will lead to repeatable and reproducible production of the coating, which to a first approximation may yield reliable performance of the coating in service. Thus, from the perspective of the entire process and application, it is clear that an integrated strategy must be considered to address supply chain reliability. The latter is a necessary, but not a sufficient step to meet the overall system goals. For instance, it has been found that during service, wide variances in performance are reported even when similar coatings are subjected to the same mechanical/environmental condition. However, it has also been anecdotally reported that there are wide variations in processing conditions, resulting in significant performance variability in coatings. Some coatings fail with very limited life (infant mortality) while others can survive through the entire engine overhaul cycle (Ref 11). Thus, it is a worthwhile endeavor to establish a baseline coating reliability through the production or repeatable and reproducible coatings through enhanced understanding of the process and coating characteristics.

\subsection{Integrated Assessment of Plasma Spray TBC Reliability: From Feedstock to a Coating}

It is well known that properties of plasma-sprayed deposits are significantly affected by the microstructure, which, in turn, is influenced by the feedstock powder, process conditions, and the resultant particle state, along with substrate and deposition conditions. Critical contributors to overall process and coating reliability can be identified as follows.

1.1.1 Feedstock Material. Most industrial TBC applications specify a rather wide particle size distribution of the yttria-stabilized zirconia (YSZ) powder for plasma spray application. They range from 10 to $75 \mu \mathrm{m}$ and follow a Gaussian distribution. Powders can be produced through different manufacturing processes which can result in 
different morphologies. The most widely used morphologies include HOSP (hollow sphere produced by plasma densification of spray dried agglomerates of fine yttria and zirconia particulates), Fused and Crushed (FC) material (obtained by melt alloying of yttria and zirconia and crushing the cast ingot and then sieving to size) and Agglomerated and Sintered (AS) material (obtained by solid-state sintering of spray-dried fine yttria and zirconia particulates). In all these cases, the key metric is the chemical alloying of the yttria and zirconia to achieve stabilization of the tetragonal structure. The fusion process assures complete and homogeneous alloying of the yttria and zirconia while the agglomeration process can introduce minor variations in composition as well as incomplete stabilization. It is worthwhile to note that significant advances in powder manufacturing processes have enabled very effective control of both particle size distribution and chemistry.

1.1.2 Spray Stream and Particle State. The plasma spray process imparts kinetic and thermal energy to the injected particles. To a first approximation, the spray stream characteristics integrate feedstock attributes, spray device configuration (nozzle, injectors etc.) and processing parameters. In the past, process control was envisaged through manipulation of the spray device and parameters as a way to control the coating. The advent of in situ, in flight particle diagnostics has allowed a more direct approach process assessment and control (Ref 12-14).

Specifically, spray stream sensors provide feedback at both the individual particle level (e.g., Tecnar DPV 2000) and characteristic process values through ensemble measurements (e.g., AccuraSpray, Spray Watch etc.). These sensors, when properly utilized will not only provide a quantitative assessment of the spray stream from the perspective measuring variability, but also allow examining effects of hardware degradation and assessment of process sensitivity to parameters. These sensors have now become part of the industrial thermal spray landscape notably from monitoring hardware degradation and process variability. They are ideally suited for assessment of temporal variations (repeatability) in production booths and, to some extent, can be used between booths and locations to assess process reproducibility.

Several studies have been reported in the literature on the linkage between particle state and process parameters, notably from the perspective of process and coating reliability. Fincke et al. discussed the scope of errors in measurement mechanism as well as algorithm of different particle state sensors, which provides an understanding of actual measurement variability associated with each particle state measurements (Ref 13). Further, Moreau and Leblanc discovered that the controlling input process parameters is not sufficient to regulate the plasma spray process as a voltage fluctuation in the power supply and wearing of electrode can change the processing conditions significantly (Ref 15). They also reported that a small perturbation in particle injection (radial for plasma spray) can lead to significant changes in particle state parameters, which eventually may alter the coating properties. Furthermore, Srinivasan et al. developed a procedure to optimize powder injection to the plasma plume through different types of commonly used particle state sensors (Ref 16). In their other study, they explored that the deposition efficiency as well as coating reproducibility improves notably with this "injection optimization" technique (Ref 17).

However, as mentioned earlier, it is important to note that particle state control is a necessary but not a sufficient condition for achieving repeatable and reproducible coatings (Ref 18). Prior study has shown that plasma spray particle streams with similar average velocity and temperature can yield coatings substantially different characters even when other parameters are constant. Particle morphology, distribution, plume character, and the method used to achieve the similar particle state all of which contribute toward the deposit formation dynamics and ensuing microstructure (Ref 19-21). This is a subject for further investigation in this article.

1.1.3 Deposition Conditions. There are several nonparticle state parameters of relevance including substrate temperature, deposition rate, spray angle, robot speed and trajectory etc., some of which can have a profound impact on the deposit formation dynamics and the ensuing microstructure. Indeed, researchers have been utilizing these attributes as ways to create novel microstructure. The segmented crack or dense vertically cracked microstructures in modern day TBCs is an example of how substrate temperatures and deposition rate can be carefully manipulated during coating microstructure.

From the perspective of process and coating reliability, these parameters have also become critical, but have received much less attention in the research literature when compared to particle state monitoring and its utilization to assess reliability.

1.1.4 Coating Characteristics Properties. Traditionally, most coating specifications call out for evaluating the microstructure of witness coupons or even selective destructive metallographic testing of real components as a measure or coating characteristics. It is widely appreciated that the technique is subjective and prone to significant testing variability. As such, there is uncertainty in utilizing microstructure as a robust measure of the three Rs. From the point of properties, hardness and bond strength are the current state-of-the-art with respect to production coating. Although, these properties are measured post spray, they are still inadequate to describe the complete coating properties.

At the design level, two main properties of interest for TBCs are: compliance and thermal conductivity in addition to failure resistances (Ref 22). Thermal conductivity is obviously important, but compliance is also critical as there is sufficient thermal expansion mismatch between the coating and substrate which can affect thermal cycling life. Compliance, to a certain extent, can be accessed through measure of coating elastic modulus. Elastic modulus for a sprayed coating is a composite measure of the intrinsic material property (in this case, YSZ in the form of splats) and extrinsic process-induced geometrical features: porosity and numerous interfaces (Ref 23). The elastic modulus is highly sensitive to feedstock material, particle state, and nonparticle state parameters and 
component attributes. Elastic modulus is a quantitative measure of the coating microstructure and can provide significant insights into coating variability (Ref 24, 25). Although used in coating design, it is rarely employed in production environments due to difficulties in measurement and interpretation of the data.

In recent years, it has further been noted that porous coatings such as TBCs are also nonlinear and anelastic solids: i.e., they cannot be defined purely by a single elastic modulus but has a dependence on stress or load. Recently, a novel approach to quantify the nonlinear and anelastic properties of the coatings through recourse to substrate curvature measurements is developed (Ref 26). Through this approach, in addition to a simplified measure of elastic modulus $(E)$, an additional parameter, namely, degree of nonlinearity (ND) in the stress-strain curve was used to quantify the microstructure along with linkage to processing conditions (Ref 27, 28).

In general, thermal conductivity is measured through laser flash techniques and generally requires thick, freestanding samples (Ref 29). Notwithstanding the geometric and measurement complexity, the technique is highly accurate. Table 1 shows the variability in the thermal conductivity measurements for the calibration sample and one plasma-sprayed coating. The variability was calculated based on nine measurements for each sample (Ref 30).

Here, when the calibration sample was measured repeatedly for nine times, errors in the range of $1.8 \%$ were observed. On the other hand, the error increases up to $5 \%$ when measurement was made on the same thermalsprayed TBC sample. Further, when a multiple specimens were prepared from the same coating, error gets further compounded resulting in almost $8 \%$ variability. The large variability in PS YSZ implies a large variation of local microstructure where the measurements were taken. Thus, the point wise measurement of thermal conductivity is not a suitable parameter to gauge coating or process reproducibility.

Based on the above discussions, understanding process and coating variability is a challenging endeavor for a stochastic process such as plasma-sprayed TBCs. It is clear that critical evaluation of reliability requires an integrated strategy; examining the variabilities in the various subprocesses and instrumentation, while simultaneously considering the interactions among the sub-processes. One approach to examine both system level and process level variabilities is through the use of "process maps" (Ref 20, $31,32)$. Of further importance are new methodologies to characterize the complex coating microstructure and property linkages. These aspects are considered in the

Table 1 Intrinsic variability in laser flash thermal conductivity measurements of plasma-sprayed YSZ coatings

\begin{tabular}{lc} 
Sample & Variability, \% \\
\hline Standard calibration sample (Pyroceram) & 1.8 \\
One PS YSZ sample & 5 \\
Six samples from same coating & 8 \\
\hline
\end{tabular}

following article. Figure 1 is an illustrative framework considered in such an integrated strategy.

\section{Experimental Procedure}

\subsection{Feedstock Materials and Characterization}

In order to investigate lot-to-lot variability in powder size distribution, three different lots (Lot 11, Lot 12, and Lot 13) of plasma-densified hollow sphere (HOSP) 204 NS powder from the same manufacturer were analyzed using Beckman Coulter LS 13320 in the dry mode. In addition, in order to check the variability in the powders from two different manufacturers (both of HOSP type), powder sample from another lot (Lot 22) was also analyzed, and the powder distribution was plotted with that of other lots (Fig. 3).

\subsection{Integrated Multi-Sensor Process Diagnostics}

For each deposition, detailed diagnostic characterization of plasma spray plume was carried out using multiple in situ sensors. For the particles and plume diagnostics, AccuraSpray g-3, DPV-2000 and SprayWatch sensors were used. All the three sensors capture information of the in-flight particle temperature and velocity ( $T$ and $V$ ) of the powder feedstock injected into the plasma. The AccuraSpray sensor uses an ensemble measuring algorithm, while DPV2000 measures individual particles. By combining both techniques, a full plume picture can be obtained, as well as assessment of each of the sensors from the point of reliability can be enabled (Ref 13).

After starting the plasma torch, the carrier gas flow rate was optimized to access the "sweet-spot" of the plume through recourse to a procedure established from earlier studies. This procedure conducted through line scan measurements of the plume, also referred to as "injection optimization" (Ref 16, 33), is a critical first step toward accurately capturing both plasma parameter effects on particle state as well as reliable diagnostics measurements. It has further been shown that an optimized injection gives you more repeatable particle state conditions, and the coating thickness (Ref 17). More details on the application of this injection optimization procedure of this study will be discussed later in this article.

DPV2000 sensor was used in "autocenter" mode to position the sensor at the maximum particle flux. At this position, for process map repeatability assessment, 5000 individual "good" particles were measured and recorded. In the case of actual coating deposition, the particle count was increased to 10,000 to have a better statistics of the particle states. For the diagnostic, a lower feed rate $(2 \mathrm{~g} /$ min) than that for actual deposition was used. Earlier, measurements were validated at a low feed rate. Although the absolute value changed, the trends in the shape of the process maps were equivalent (Ref 34). Figure 2 presents a schematic of the layout of all the sensors generally used for a plasma spray process. Though, only DPV2000 and AccuraSpray sensors are reported in this study, for the 


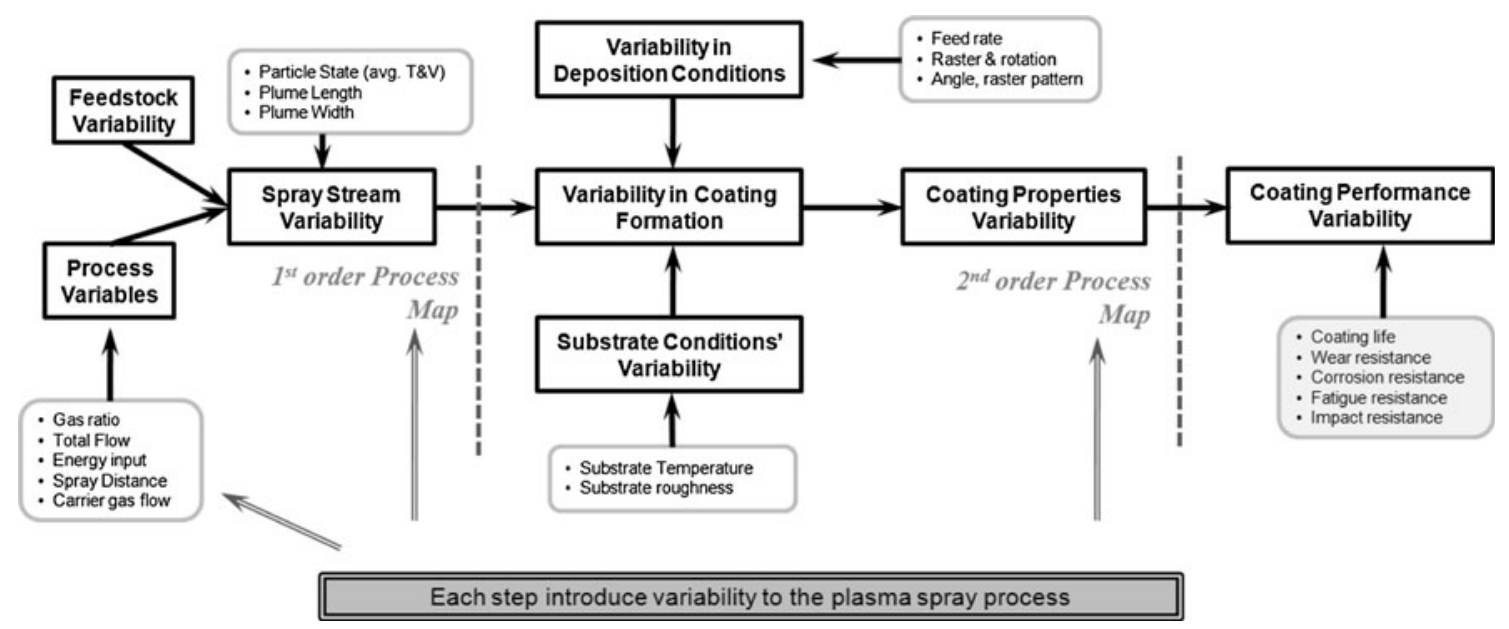

Fig. 1 An illustrative overview of integrated assessment of variabilities associated with the thermal spray process

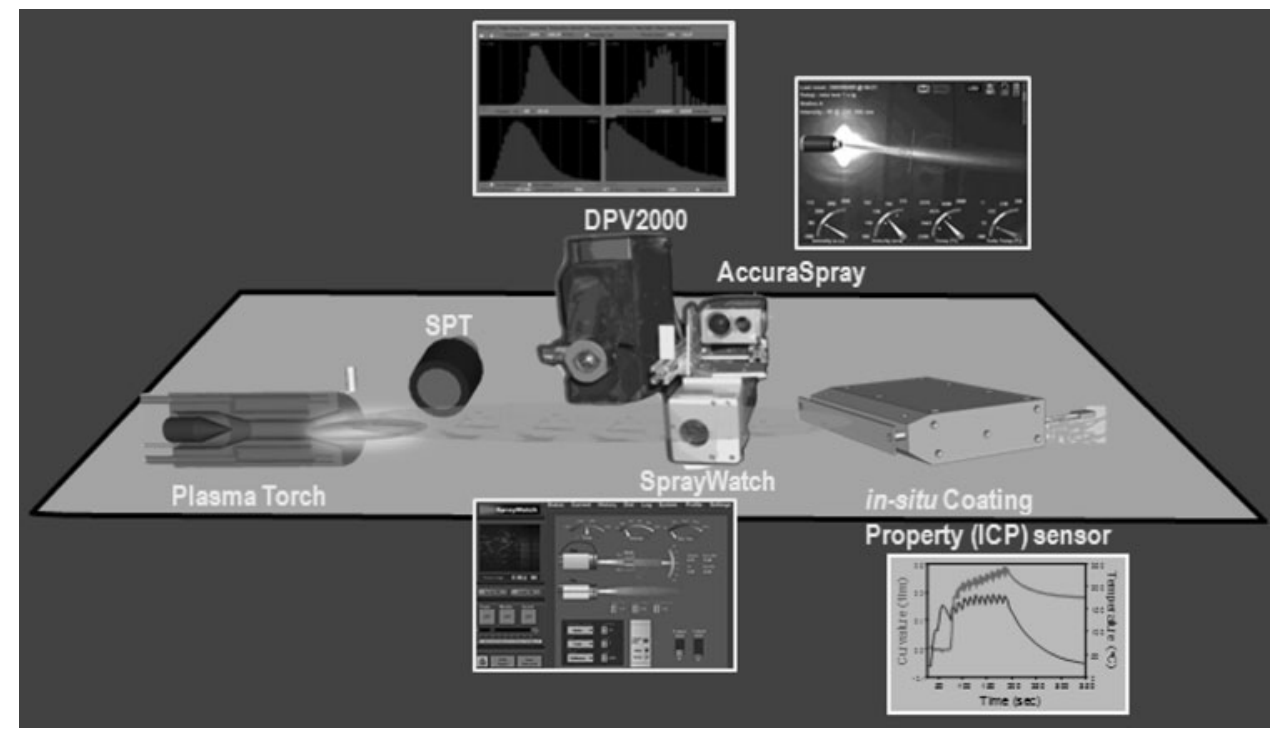

Fig. 2 Illustration of the 3D multi-sensor particle measurement set-up along with in situ coating property characterization

sake of having broader inspection of a process, some other sensors are also shown in the figure.

\subsection{Monitoring Coating Deposition}

2.3.1 Repeatability of the APS Process Space: FirstOrder Process Maps. Twelve temperature and velocity ( $T$ and $V$ ) conditions were selected over the wide range of a first-order process map. These conditions were selected based on a central composite design-of-experiment (DoE) strategy. Condition 1 is nominally the center condition of the process map, representing typical process parameters for depositing coatings (e.g., this is used as the standard parameter at the Center for Thermal Spray Research for routine coating preparation). For each of 12 points in the process map, the center condition (condition 1) was repeated four times equally spaced during the entire measurement. The experiments were performed using a Sulzer Metco F4- $\mathrm{H}_{2}$ swirl flow plasma torch. The spray plume and particle diagnostic was carried out using the procedure mentioned in Sect. 2.2. Partially stabilized YSZ HOSP powder of standard commercial grade (Lot 11 in Fig. 3) was used for the diagnostics experiment as well as deposition. The process maps were created over a period of several days and multiple hours of plasma torch time. This time span represents a measure of process stability in a controlled academic setting to examine intrinsic variabilities rather than a measure of production variability.

This strategy of diagnostics was applied for all the 12 conditions. Table 2 provides the absolute values as well as the range of arc current, argon, and hydrogen flow rates of the 12 conditions. These parameters were determined after iterative calculations required for obtaining certain $T$ and $V$. For all the experiments, the nozzle diameter was 


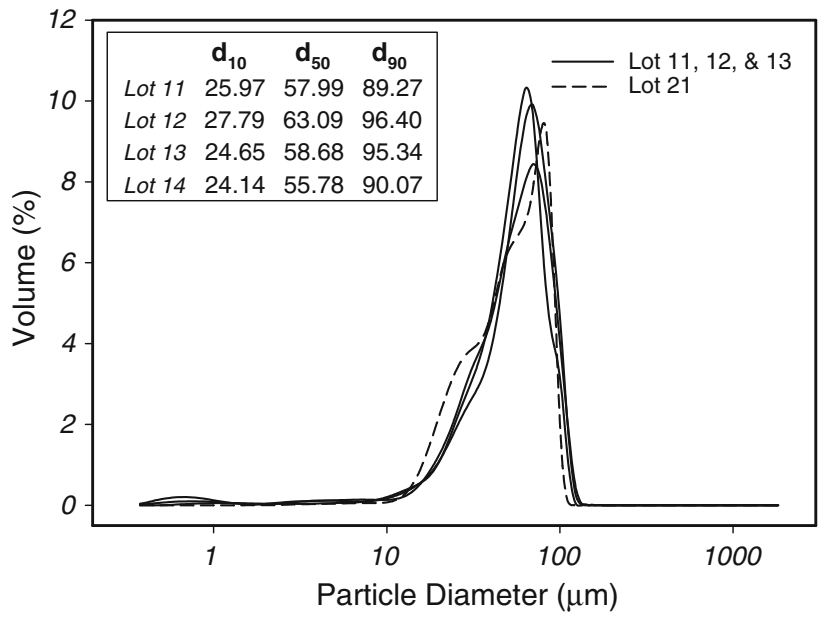

Fig. 3 Lot-to-lot variability in HOSP powder size distribution from a single manufacturer (Lots 11, 12, and 13) and a different manufacturer (Lot 21)

Table 2 Process parameters used for design of experiment performed over 5 consecutive days to examine process repeatability of 12 different conditions

\begin{tabular}{lccc}
\hline Condition & Argon, sl/m & Hydrogen, sl/m & Current, A \\
\hline 1 & 47.5 & 6.0 & 550 \\
2 & 35.0 & 6.5 & 450 \\
3 & 60.1 & 4.4 & 650 \\
4 & 47.5 & 6.0 & 550 \\
5 & 59.7 & 11.1 & 450 \\
6 & 59.7 & 11.1 & 650 \\
7 & 35.2 & 2.6 & 450 \\
8 & 47.5 & 6.0 & 550 \\
9 & 35.2 & 2.6 & 650 \\
10 & 60.1 & 4.4 & 450 \\
11 & 35.0 & 6.5 & 650 \\
12 & 47.5 & 6.0 & 550 \\
Max & 60.1 & 11.1 & 650 \\
Min & 35.0 & 2.6 & 450 \\
Range & 25.1 & 8.5 & 200
\end{tabular}

An iterative algorithm was used for selecting these parameters to have corresponding $T$ and $V$

$8 \mathrm{~mm}$. The powder injector was at an offset of $7 \mathrm{~mm}$ vertically upward from the nozzle axis, and the angle of injection was perpendicular to the nozzle axis. Although both single particle (DPV2000) and ensemble (AccuraSpray) measurements were carried out, only DPV2000 measurements are included here. (Both sensors showed similar variability, but the AccuraSpray did not yield sufficient fidelity in the data for robust comparisons.) The data collected was used to generate a first-order process map by making a $2 \mathrm{D}$ plot of the average temperature and velocity of each condition. This scan of 12 -process map conditions was performed five times each over a course of one week. These results are discussed in Sect. 3.3.

2.3.2 Coatings Repeatability Assessment Studies. 2.3.2.1. Deposition: After analyzing the data obtained by process map repeatability experiment, a set of

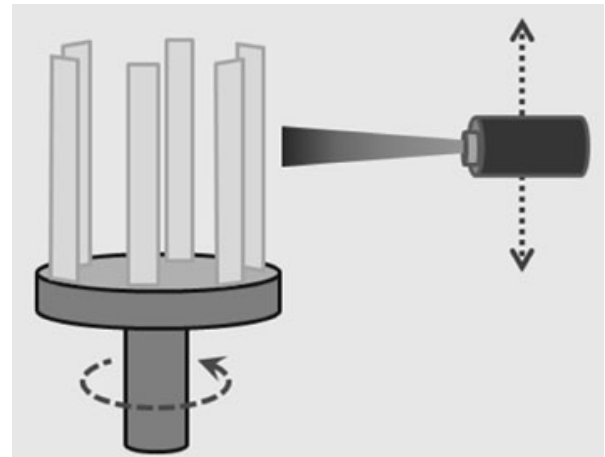

Fig. 4 Schematics of the carousel set-up for multiple sample fabrication in a single spray run

experiments was designed to measure the variability of coating properties sprayed at the center condition of the first-order map. Three batches of coatings were sprayed over different time spans (each batch sprayed within a week). All coatings were made on $9^{\prime \prime} \times 1^{\prime \prime} \times 1 / 8^{\prime \prime} \mathrm{Al} 6061$ substrates. The substrate was selected based on our ability to conduct both in situ and ex situ curvature measurements. The substrates were grit blasted using $60-\mu \mathrm{m}$ silicon oxide grit with a pressure of 55-60 psi, and the substrate surfaces were cleaned by compressed air (40 psi). The details of these batches are given below:

Batch 1: These individual coating runs tied to the process map experiments. They were conducted over a span of 5 consecutive days. In-flight particle diagnostics was performed using DPV2000 sensor before each deposition. Coatings were deposited on the ICP sensor, which allows simultaneous measurement of substrate curvature and substrate temperature $(\operatorname{Ref} 35,36)$. This approach allows extraction of deposit stress evolution during deposition as well as elastic properties of the coating. During the coating deposition, the temperature of the substrate and coating were controlled by providing a continuous supply of compressed air (40 psi) via an array of nozzles in the ICP sensor as well as two air jets attached sideways to the plasma torch directing toward the coating. After the deposition, the coated specimen was cooled only by the ICP sensor's air jets. The coating-substrate temperature was adjusted by regulating the compressed air pressure and was monitored by two contact thermocouples (Omega Inc.) in contact with the substrate (Ref 35).

Batches 2\&3: Several coatings were prepared in a single experiment by spraying onto a carousel arrangement. Four samples were mounted vertically and spun perpendicular to the spray axis with an angular speed and robot raster speed of $72 \mathrm{rpm}$ and $2 \mathrm{~m} / \mathrm{s}$, correspondingly. The plasma torch was rastered vertically (Fig. 4). Again, in-flight particle diagnostics was conducted using DPV2000 sensor before performing actual spraying. In addition, for the batch 3 , substrates were pre-annealed for $2 \mathrm{~h}$ at $350{ }^{\circ} \mathrm{C}$ to eliminate any residual stresses due to grit blasting, and a tighter powder cut was used for deposition than that used for batches 1 and 2. The powder size distribution curves for these batches are shown in Fig. 5. In this case, the 


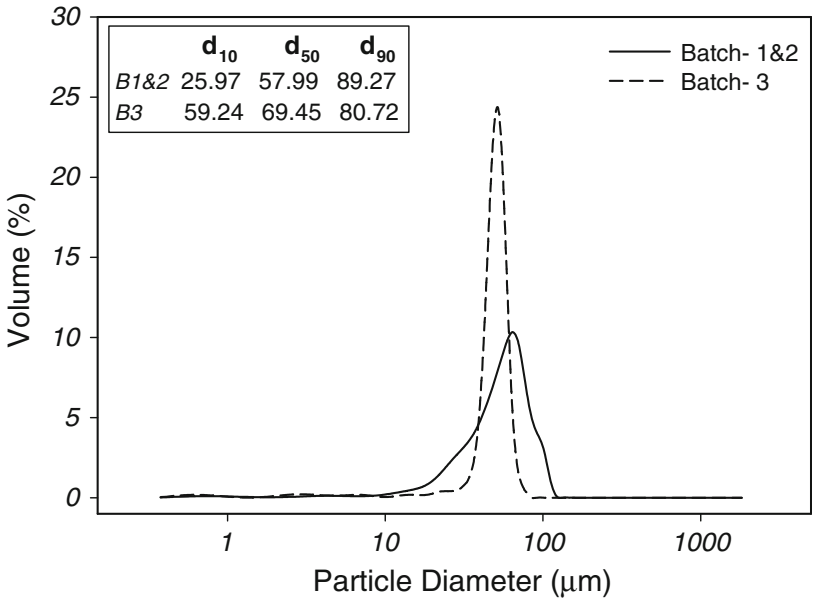

Fig. 5 Distribution of powder sizes used in the fabrication of coatings

substrates temperatures were not monitored, and the specimens were cooled on the spinning table under ambient conditions.

For all the deposition experiments, HOSP powder was used, and the aforementioned diagnostic strategy was used except for the number of particle sampled (in this case 10,000 particles) for DPV2000 measurements. A micrometer (Mitutoyo Corp.-S/N 293-344) with a resolution of $1 \mu \mathrm{m}$ and weighing instrument (Fisher ScientificA200DS) with a resolution of $1 \mathrm{mg}$ were used for thickness and weight measurement, correspondingly. For both coating thickness as well as coating weight calculations, corresponding measurements of substrate were subtracted from that of the deposited beam.

\subsubsection{Thermal Cycling Using Ex Situ Coating Prop-} erty [ECP] Sensor: After spraying of the three batches of carousel, the post-deposition residual curvatures of the coated specimens were measured using dial depth micrometer (Compac Geneve) with a resolution of $1 \mu \mathrm{m}$. The specimens were then thermally cycled using ex situ curvature sensor, referred to as ECP, for ex situ coating property monitoring. In this version of ECP setup, the specimen is heated inside a muffle furnace (ThermolyneF6018) to provide a uniform heating and cooling with a through thickness temperature difference of $1-2{ }^{\circ} \mathrm{C}$. For a heat cycle, the specimen is mounted vertically on ECP sensor; it is then heated up to $250^{\circ} \mathrm{C}$ and is cooled down back to the room temperature. Typically, this process takes $30 \mathrm{~min}$ for heating from room temperature to the maximum temperature, and approximately $10 \mathrm{~h}$ for cooling. In order to shorten the cooling time, the specimen is taken out of the furnace at around $180{ }^{\circ} \mathrm{C}$ to cool down in the ambient condition. A self-adhesive thermocouple (SA1XL-K-72, Omega Engineering Inc.) is glued on the substrate side of specimen for accurate measure of the temperature. For each specimen, at least three heat cycles were made to get repeatable curvature-temperature behavior. For each case, second and third cycles were found repeatable; the second cycle was considered for further analysis.

\subsubsection{Nonlinear Properties Extraction from Curva-} ture-Temperature Data Obtained from Heat Cycling: The curvature-temperature plots for each specimen shows a nonlinear behavior with some hysteresis between the heating and cooling cycles. The reason behind this nonlinear and hysteresis behavior has been discussed in detail in earlier studies ( $\operatorname{Ref} 26,28)$. The behavior at the beginning of cooling highly depends on the maximum temperature during heating (Ref 28). Thus, interpretation of cooling curves must be made in conjunction with the maximum temperature of thermal cycle. Since the maximum temperature varies slightly for each day, only the heating curve is used to interpret the measurement.

A detailed description of the coating's anelastic behavior is also described in a previous study (Ref 26). The referred study discusses the steps to calculate the nonlinear elastic relations from the low temperature thermal cycle data using a phenomenological constitutive uniaxial stress-strain model. After processing the curvature-temperature data via an inverse analysis technique, required material parameters are estimated to describe the complete stress-strain relation of the coating. For the sake of consistency, the measurements in the temperature range of $T_{\text {init }}=\sim 30{ }^{\circ} \mathrm{C}$ and $T_{\max }=\sim 200{ }^{\circ} \mathrm{C}$ are used in all the specimens. Although the inverse analysis identifies five relevant variables, they are the processes to determine two key parameters: one is the Young's modulus at the room temperature $E$, and the other is the nonlinear degree (ND), which quantifies the nonlinear behavior of a coating. Since each coating has its own unique stress-strain behavior resulting from its overall microstructures, these two parameters represent a novel quantitative measure of coating properties. Liu et al. in an earlier study represented this in the form of a $E$ versus ND map and demonstrated that this approach can be readily linked to coating microstructure and its sensitivity to process condition (Ref 27).

2.3.2.4. Thermal Conductivity Measurements: In order to prepare samples for thermal conductivity measurements, a sample of dimension $15 \times 25.4 \mathrm{~mm}$ was cut from each beam. The substrates in each piece were ground to a thickness 100-300 $\mu \mathrm{m}$. The free standing coating samples were then detached from their remaining substrates, and were rounded to the disks with diameter of approximately $12.7 \mathrm{~mm}$ and coated with a very thin graphite layer using a Acheson-Aerodag G spray-can. The thermal conductivity was measured by Holometrix Laser Flash Instrument at room temperature. Each sample was subjected to a short laser pulse, and corresponding temperature rise was measured on opposite side. The specific heat was measured first for a calibration sample, and then for the free standing specimens. With an additional input of density and thickness to the software, thermal conductivity of different samples was calculated. 
2.3.2.5. Elastic Modulus and Hardness by Micro Indentation Method: A small piece of the coated specimen with dimension $5 \times 7 \mathrm{~mm}$ was cut from the curvature beam. The top surface of coating was polished up to a finish of $3 \mu \mathrm{m}$. Depth-sensing indentation method was used to extract the materials properties using the contact response of a small volume of material. Nanotest 600* (Micro Materials Limited, Wrexham Technology Park, Wrexham, LL 137YP, UK) instrument was used to perform the experiments. In this study, a $1.59-\mathrm{mm}$ diameter WC-Co spherical indenter was used with a maximum load of $5 \mathrm{~N}$. The maximum loads of each cycle are equally divided between zero and the total maximum load, with a partial unloading to $75 \%$ of the maximum load being used. Oliver and Pharr method was used to analyze the load-displacement data. For each samples, 10 indents were made, and the average value as well as standard deviation of elastic modulus and hardness were noted.

\section{Results and Discussion}

\subsection{Measure of Feedstock Variability}

Although in this study, a single lot of standard powder was used for most of the spray runs to process and coating variability, it is worthwhile to consider lot-to-lot powder variations from the point of system level reliability. Detailed assessment of such variability is beyond the scope of this article, but it is worth noting that three lots of powders obtained over a three-year period from the same manufacturer shows very similar distributions (Fig. 3). However, as expected, there are some variations when two different manufacturers' powders are used.

\subsection{Particle State Reliability}

3.2.1 First-Order Process Maps and Assessment of Particle State Variability in a Single Run. The advent of in situ particle diagnostics has allowed rapid evaluation of the plasma spray process parameters on the particle state. To the first approximation, particle temperature and particle velocity capture the state of spray stream. The $T$ - $V$ diagram with appropriate linkages to parametric effects is referred to as the first-order process map for plasma spray. The map can also be used to assess particle state variabilities within the context of the observed process range of the measured responses. Figure 6 shows the first-order map derived from a 44-measurement set-up through a central composite design-of-experiment strategy. Figure 6 also overlays trend as to how different particle states are associated with change in process parameters. Quantitative effects can also be derived from such maps as was depicted in Ref 20. The parameters varied were primary flow, secondary flow, and gun current, with particle states being monitored through DPV 2000. The parametric modifications provided an operational space of $301{ }^{\circ} \mathrm{C}$ in particle temperature and $78 \mathrm{~m} / \mathrm{s}$ in particle velocity. For each of the conditions, the injection conditions were optimized so as to subject the particle to the same

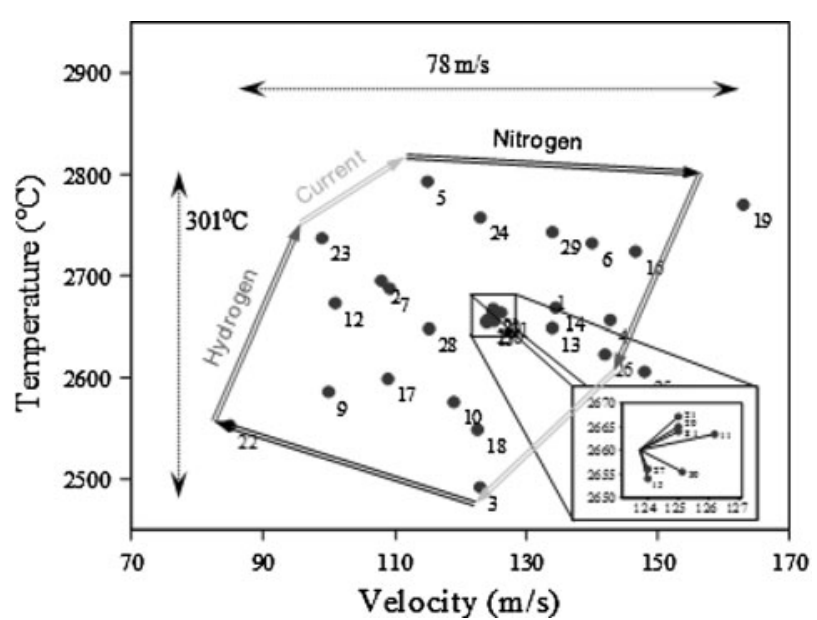

Fig. 6 First-order process maps representing the influence of key plasma torch parameters on average particle temperature and velocities (data collected from a central composite design of experiments). The design of experiments allowed for repeated measurements at the center condition enabling assessment of intrinsic variability in particle state for a single run

measurement volume in the plume. Referred to as the "sweet-spot," this approach is described in detail in Ref 18 and 20 and is an important first step not only for achieving maximum energy transfer between process and particles but also for reliable measurement of particle states. In other words, this allows for a true comparison of the particle-plasma interaction for a given parameter set.

Relevant here is the repeated measurement of the "center" condition of the central composite design which allows assessment of variability in particle state for a single run. This is depicted in the inset of Fig. 6. The center condition was repeated approximately seven times and based on these measurements, it is determined that average variability in temperature is $13.3^{\circ} \mathrm{C}$, while the average variability in velocity is $2.2 \mathrm{~m} / \mathrm{s}$. Normalizing these numbers with respect to the measured range in the first-order map (rather than the absolute value of temperature velocity will allow a more realistic assessment of the particle state variability) yields $\Delta T / T_{\text {ave }} \sim 4.4 \%$ and $\Delta V /$ $V_{\text {ave }} \sim 2.8 \%$.

The above measurements were for the FC YSZ powder. Hollow spherical (HOSP) powder displays a somewhat higher scatter in both temperature and velocity $\left(61{ }^{\circ} \mathrm{C}\right.$ and $\left.11 \mathrm{~m} / \mathrm{s}\right)$, while the scatter in solid spherical (AS) is between that of FC and HOSP $\left(37^{\circ} \mathrm{C}\right.$ and $3.5 \mathrm{~m} /$ s). The exact reason for the increased scatter for the HOSP powder is not clear. It may be related to lower density of the particle and varying degrees of hollowness among the particles (Ref 19).

This estimate of the total error includes contributions from

- Instrumental errors of the DPV 2000

- Control errors to establish the individual spray conditions, namely, the control of primary/secondary/ carrier gas flows, amperage, and feed rate 
- Degradation effects during the experimental time of $3.5 \mathrm{~h}$, namely, nozzle, cathode, and injector wear (given that these results were obtained from a single run, it is likely that there is minimal torch degradation during this period-the run was $3 \mathrm{~h}$ and 26 min long). Furthermore, uncertainties with respect to start-stop, measurement positioning etc. are reduced allowing the best possible assessment of achievable particle state variability in plasma spray).

These results provide a baseline assessment of particle state variability. The implications of reliability of particle state have been assessed at the different stages in the process, and is presented elsewhere (Ref 17). Typically these errors are accentuated during sustained operation of the torch over extended periods. In this study, all the three aforementioned morphologies were studied with a new nozzle and subjected to nominally short duration of process times.

3.2.2 Comparisons of Different Particle Sensors in a Single Run. In the above experiment, only DPV 2000 was used to measure particle properties. Since DPV 2000 measures individual particle properties, each average is a composite measure of some 5000 particles and, as such, provides high degree reliability (although particle size distribution effects are also captured here). Most industries have resorted to the use of characteristic process sensors, such as AccuraSpray, SprayWatch etc., which provide an ensemble measurement of the entire plume at any given instant. As such, it is important to establish a baseline distinction among the three sensors.

Table 3 provides a comparative analysis of the three sensors, again measured during a single long spray run $(3 \mathrm{~h})$ by repeatedly moving the torch among the sensors and recording the data. Here, only a single spray parameter was considered. The results suggest that reported values of $T$ and $V$ were different even for the same process condition $(T$ and $V)$, which is a result of different measurement volumes and extraction algorithms associated with different sensors. However, comparing each sensor within itself allows assessment of intrinsic variability specific to respective sensor. For instance, since DPV utilizes individual particle measurement, they average over a much larger population. However, DPV also captures a larger range of particle properties associated with differences in size and position. AccuraSpray takes a single snapshot of the plume and provides a characteristic value of the spray stream. As such, its average is reported from a

Table 3 Assessment of variability in particle state measurements by different sensors over a 2 -h run

\begin{tabular}{|c|c|c|c|c|c|c|}
\hline \multirow[b]{2}{*}{ Sensor } & \multicolumn{3}{|c|}{ Temperature $[T],{ }^{\circ} \mathrm{C}$} & \multicolumn{3}{|c|}{ Velocity $[V], \mathbf{m} / \mathbf{s}$} \\
\hline & Average (max & $\begin{array}{l}\Delta T \\
x-\min )\end{array}$ & SD & Average & $\begin{array}{c}\Delta V \\
(\max -\min )\end{array}$ & SD \\
\hline DPV 2000 & 2989 & 17 & 4.7 & 141 & 4.5 & 1.4 \\
\hline AccuraSpray & 2654 & 9 & 3.2 & 164 & 1.5 & 0.5 \\
\hline SprayWatch & 2685 & 14 & 4.4 & 149 & 5.7 & 1.8 \\
\hline
\end{tabular}

few measurements over the same time domain. SprayWatch is a camera-based technique with a different view field and, thus, represents a third set of measurements. However, one can conclude from all the three measurements that there is limited intrinsic particle state variability for a single experimental run (without gun start stop) and provides a baseline for comparison of temporal and spatial variations.

\subsection{Repeatability of the Process Space: Comparison of Five First-Order Process Maps}

In the experimental study discussed above, the variabilities in particle state were considered only for a single parameter presented within the context of the process map. The results suggest that the intrinsic variability of plasma spray particle state is surprisingly small, at least, for a single run measurement. However, the intrinsic variability may also have a dependence on the type of process parameters used to access a given particle state. The process map approach allows us to critically examine this variability.

In order to assess the variability across the process space, the entire first-order process map was repeated five times over many days. For each experiment, the torch was turned on only once, and the particle injection was optimized to the said "sweet-spot" of the spray torch. Thus, although each process space was reasonably reproducible, the study allows examination of the torch start-stop effects on the process space repeatability. The results of five first-order process map experiments are shown in Fig. 7. Figure 7(a) displays the average value of the particle $T$ and $V$ obtained for each condition, while Fig. 7(b) represents the same data in terms of scatter bar with twosigma variation.

Several important conclusions are self-evident from this study. Condition 4,8 , and 12 fell in the vicinity of center condition (C1) on process map. Therefore, only nine conditions are visible on the process map. It can clearly be seen that the variability of different conditions in the process map depends on the location of that condition within the process space. For example, on the one hand, condition 2 (C2) having lower velocity of particles shows the largest variability; on the other hand, condition 5 (C5) exhibits the tightest $T$ and $V$ distribution among all the points. These two conditions are the extreme conditions in terms of variability for this particular process map. The center condition, typically used to produce coatings at CTSR Stony Brook, lies somewhere in between $\mathrm{C} 2$ and $\mathrm{C} 5$.

Apart from the average $T$ and $V$ values, obtained from DPV2000 sensor response, the analysis of the distributions reveal other interesting attributes about the particle spray stream that are not prominent by just examining the averages (Ref 19, 37). For instance, a spray condition may result in the same average $T$ and $V$, but it may result from different distributions of data. A detailed analysis on the correlation of average $T$ and $V$ with the distribution has been discussed in Ref 37. It has been shown that, at least in the case of YSZ, the distribution provides insight into

\section{.}


extent of melting. As such, even though the difference in the average values of $T$ and $V$ may be small, the effect of melting state can be significant with concomitant effects on coating properties.

Detailed analysis of the distributions has been conducted for this particular set of experiments (Ref 38), but it is somewhat too exhaustive to be presented in this article. An alternative approach to capture the effects of distributions is through the use of group parameters to describe particle states. Earlier study has provided a framework to define the relevant group parameters to describe the particle thermal state and particle kinetic state. The particle melting index (MI) captures the thermal state of the particle by normalizing the temperature with respect to the dwell time and size (Ref 39, 40). On the other hand, kinetic energy (KE) is an excellent descriptor of the particle kinetic state as it includes the size information through mass measurements, and the velocity is qualified as a square function to capture the energetic (Ref 33). These results are described in Fig. 8.

Comparison of superficial in-flight particle properties temperature and velocity to grouped parameters MI and $\mathrm{KE}$ leads to some interesting observations (Fig. 8). For most conditions, the percent deviation of average MI-KE was found to be much larger than that of average TV (Fig. 7). One explanation for this is that the additive combination of variability that occurs when calculating MI using both temperature and velocity measurements. The calculation of KE contains a velocity-squared term which compounds the velocity deviation increasing $\mathrm{KE}$ variability. The small average $T$ - $V$ variability of condition five was seen to carry over into MI-KE space where it had the smallest MI variability and low KE variability. Although condition two had the largest distribution in TV space, other conditions in MI-KE space were found to have larger MI-KE variability, i.e., conditions 7 and 11.

While average $T-V$ and average MI-KE mask some of the subtleties of the spray stream characteristics, more information can be extracted when particle state distributions are analyzed. When histograms of the tight, center, and wide conditions were made, a link between the MI distributions and the average MI variability was found (Fig. 9). Condition 5, which had the lowest average $T-V$ variability, had very consistent MI distribution shapes throughout the five spray runs. In contrast, condition 2 with the highest average TV variability was seen to demonstrate significantly varying MI distribution shapes between spray runs.
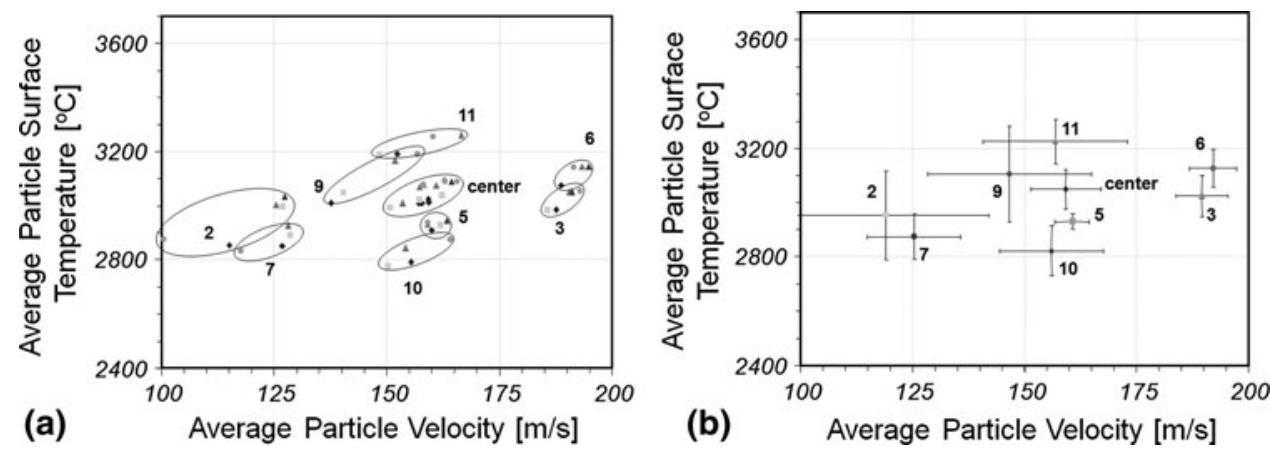

Fig. 7 Parameter specific variability in particle states obtained by comparing first-order process maps constructed over 5 days. (a) Range of average $T$ and $V$ data for each point measured from DPV 2000; (b) Represented with 2-sigma standard deviation for each condition

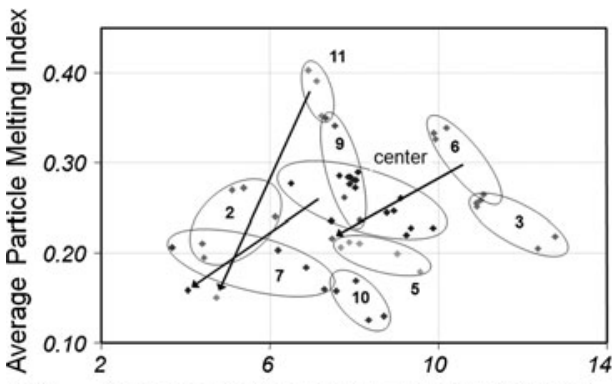

(a) Average Particle Kinetic Energy $\left(10^{-7}\right)[\mathrm{J}]$

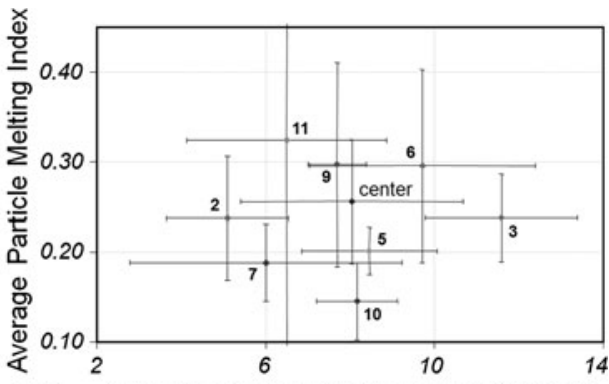

(b) Average Particle Kinetic Energy $\left(10^{-7}\right)[\mathrm{J}]$

Fig. 8 Melting index vs. kinetic energy plots for repeated process maps calculated from DPV2000 data (a) distributions of average MI-KE for each condition over the five process maps with circles containing points within the same condition (arrows point to stray points from that condition), (b) simplified representation showing averaged MI-KE and $2 \sigma$ standard deviation error bars 


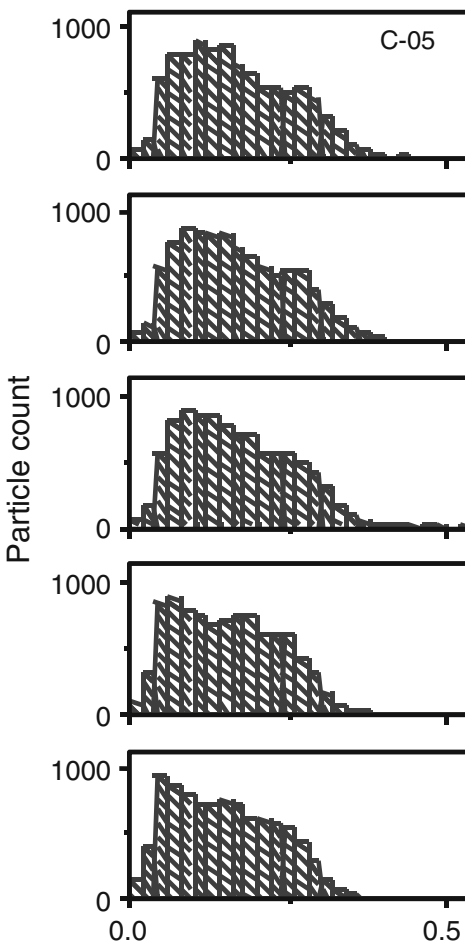

(a)
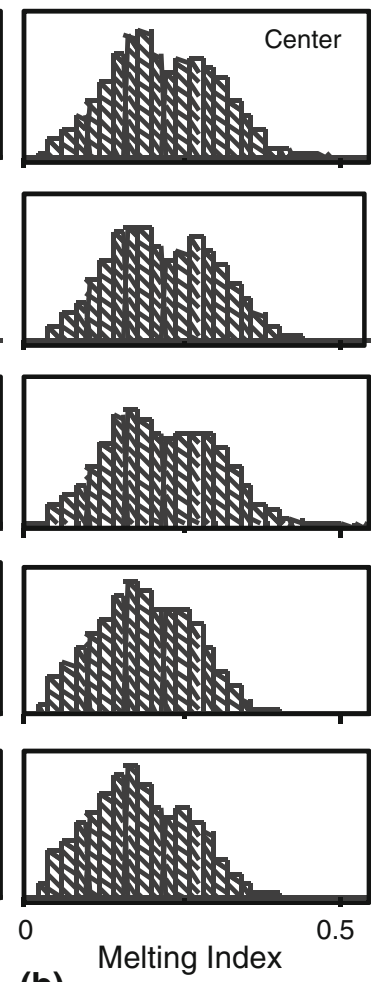

(b)
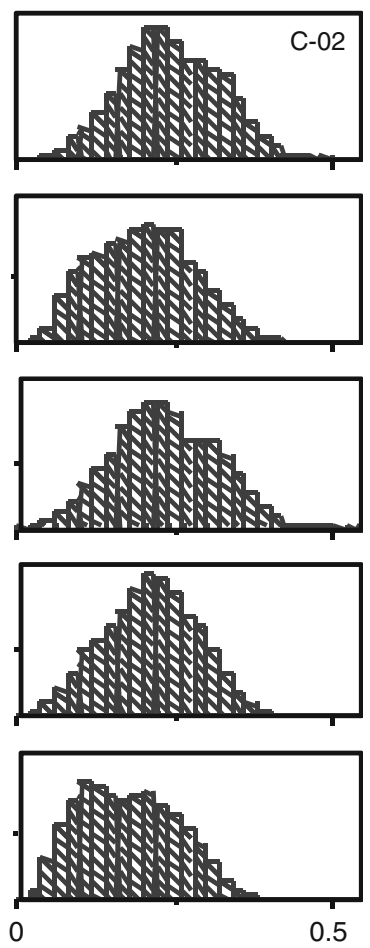

(c)

Fig. 9 Melting index distributions for the (a) tight, (b) center, and (c) wide spray conditions in TV space (C5, Center condition, and C2, respectively) over 5 days

\subsection{Repeatability of the Center Condition of the Process Map: Particle State and Coating Properties}

3.4.1 Particle State Evaluation. The center condition from the above process map was used to produce one coating each over the same 5 days of the variability study. All the coatings were fabricated on the ICP sensor and also analyzed in detail through ex situ characterization. In this situation, the torch input parameters (argon flow rate, hydrogen flow rate, and current) were fixed. Ensemble and individual particle in-flight diagnostics were taken immediately before each coating deposition by the AccuraSpray and DPV2000 sensors. From both sets of data, the average $T-V$ variability was calculated. The $T-V$ distribution variability was also examined using the individual particle data from the DPV2000. In the latter case, a total of 10,000 particles were monitored prior to each ICP run. The individual particle data set was also used to calculate MI and KE for each run and their respective averages were determined (Table 4). It is clear from the results that the variability in $T$ and $V$ values measured in different days was only marginally higher than those reported during single long spray run (17 degrees versus 23 degrees) in Table 3 . This is partly attributed to the injection optimization procedure which has been shown to substantially reduce day-to-day variability in particle state (Ref 17).
Table 4 Variability in particle temperature, velocity, MI, and KE of the center condition obtained from five experiments conducted over 5 days

\begin{tabular}{lccc}
\hline Property & Average & Range (max - min) & SD \\
\hline$T,{ }^{\circ} \mathrm{C}$ & 3085 & 23 & 11 \\
$V, \mathrm{~m} / \mathrm{s}$ & 164 & 9 & 4 \\
Melting index & 0.28 & 0.01 & 0.005 \\
Kinetic energy $\left[\times 10^{-7}\right], \mathrm{J}$ & 7.39 & 0.41 & 0.18 \\
\hline
\end{tabular}

3.4.2 Coating Deposition and In Situ Coating Property Evaluation. Five coatings (batch 1) were deposited over as many days on a $9^{\prime \prime} \times 1^{\prime \prime} \times 0.125^{\prime \prime} 6061-\mathrm{T} 6$ aluminum substrate while simultaneously recording substrate temperature and the substrate curvature through the ICP sensor. The feed rate for the one out of five was not same as the latter four (thickness per pass optimization was too high on the first day); hence, was not further examined in this week-long repeatability study. For the remaining samples processed using identical procedures, the evolving stress, thermal stress, residual stress, and elastic modulus were calculated, and are tabulated in Table 5.

At the first observation, it is clear that the in situ stress evolution and build-up during deposition were not the same for the four specimens. Though, the evolving stresses, which develop due to quenching of molten particle as they get deposited on the substrate, were quite similar

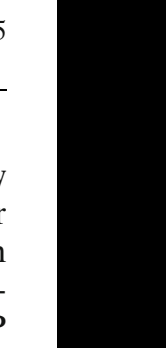

$x^{2}$

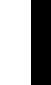
.

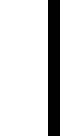

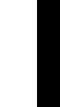

(1)

(n)

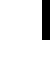


Table 5 Coating properties from samples obtained from repeated center conditions sprayed over 4 days

\begin{tabular}{lcccc}
\hline $\begin{array}{l}\text { Sample } \\
\text { no. }\end{array}$ & $\begin{array}{c}\text { Thickness } \\
\text { per pass, } \\
\boldsymbol{\mu m}\end{array}$ & $\begin{array}{c}\text { Evolving } \\
\text { stress, } \\
\mathbf{M P a}\end{array}$ & $\begin{array}{c}\text { Thermal } \\
\text { stress, } \\
\mathbf{M P a}\end{array}$ & $\begin{array}{c}\text { Residual } \\
\text { stress, } \\
\mathbf{M P a}\end{array}$ \\
\hline $\mathrm{B} 11$ & 34.40 & 17.4 & -38.1 & -30.6 \\
$\mathrm{~B} 12$ & 31.40 & 14.1 & -45.7 & -33.3 \\
$\mathrm{~B} 13$ & 32.86 & 14.4 & -47.4 & -28.1 \\
$\mathrm{~B} 14$ & 32.53 & 18.8 & -49.7 & -34.8 \\
\hline
\end{tabular}

with the maximum difference of $4.4 \mathrm{MPa}$, the thermal stresses due to mismatch in coefficient of thermal expansion (CTE) were somewhat different (11.6 MPa). Here, the positive values of evolving stresses suggest the dominance of quenching over peening for all of the coatings, which is generally observed in the case of YSZ powders. It is unclear whether the $11.6 \mathrm{MPa}$ is of significance and would require additional data for further quantification through nonlinear elastic analysis schemes described in the next section. However, the number does provide a benchmark for quantitative assessment of typical production variability within the booth.

3.4.3 Post-Spraying Coating Properties Evaluation. Subsequent to plasma spraying, the thicknesses and weights of each sample were recorded and compared (the thickness was measured at five locations across the beam and averaged). The nonlinear analysis of the four coatings was performed via thermal cycling using the ECP sensor. Further, since, sample preparation for microstructure, thermal conductivity, and elastic modulus (as well as hardness) requires cutting of a sample, these properties were measured for three randomly chosen samples only, and one sample was kept for future reference. Each of these ex situ techniques provides information on certain aspects of the coating microstructure. The curvaturederived modulus and nonlinear degree provide information globally in plane properties of the coatings, including overall porosity. On the other hand, micro-indentation technique to some extent provides information on the local coating properties. In addition, the thermal conductivity is a comparative measure of the coatings interlamellar bonding and porosity (Ref 41). The integrated understanding from these properties can be presented as a comprehensive characterization of the coating. An exhaustive study on all these coating property characterization techniques has been conducted in the past, and, therefore, the current study concentrates on the variability among the coatings rather than on the physics behind their measurement techniques.

The results for batch 1 are included in a subsequent Sect. 3.5 along with those for batch 2 and batch 3 enabling a comprehensive comparison of variability among deposition strategies.

\subsection{Coatings Repeatability: Multiple Coatings for Single Process Condition}

The above study examines temporal variations of the spray process and the ensuing coating properties through an integrated study of both particle state and deposit state. The temporal variations were assessed for a relatively short testing period of about 5 days. No significant degradation of hardware was expected during this very limited usage of the system and as such the effort was targeted more toward establishing torch start-stop-related variability.

A second study was conducted to examine variability among several coatings all prepared during a single spray run. The goal here was to examine the intrinsic variability in coating properties without introduction of any spray stream variability. In order to accomplish this goal, two sets of experiments were conducted through YSZ deposition on a rotating carousel. Details of the test have been provided in Sect. 2.3.2.1.

Since batch 2 and batch 3 coatings were sprayed during a single spray run, and could not be made on ICP, information on stress evolution was not available. However, particle states were monitored prior to the spray run, and all coatings were post spray characterized consistent with those performed in batch 1 . Figure 10 presents a comparative assessment of different coating properties from the same batch as well as those from different batches. The absolute values of the numbers obtained from different characterizations are perhaps not readily comparable; however, relative comparison of variability among the various sets of coatings can be made to shed light on the process. The error bars shown in the figure are the representation of variability in the measurement made on a single specimen repeatedly.

Figure 10(a) shows thickness numbers of the three batches. As expected, batch 1 shows more variability in thickness as it was sprayed over many days, while batch 2 and batch 3 show reasonably repeatable coating thicknesses resulting from a single spray run onto the carousel-mounted specimen. There is a small difference in the degrees of variability in batch 2 and batch 3 , which is attributed to different powder cuts used for fabrication of the coatings (For batch 3, a finer powder cut was investigated to examine the effect of particle size distribution on variability). Variability in densities (Fig. 10b) in the coatings from a same batch as well as different batches is less than the error associated with the measurements, and is not sufficiently significant for comparison.

Thermal conductivity data from Fig. 10(c) suggests that coatings have significant variabilities in their thermal conductivity. However, this may be related to the intrinsic variability in the measurement techniques (Table 1). The error bars shown in the figure are based on $5 \%$ variability number which was calculated from the measurement made on a typical PS-YSZ coating several times. It is unclear at this stage whether any conclusion can be drawn on the intrinsic variability of the coating thermal conductivity. Improved measurement techniques with low testing variability will be required for sorting out the coating attributes.

The elastic modulus and hardness numbers (Fig. 10e, f) for the various coatings obtained from micro-indentation technique appear different suggesting some local variability among the coatings associated within each batch. 

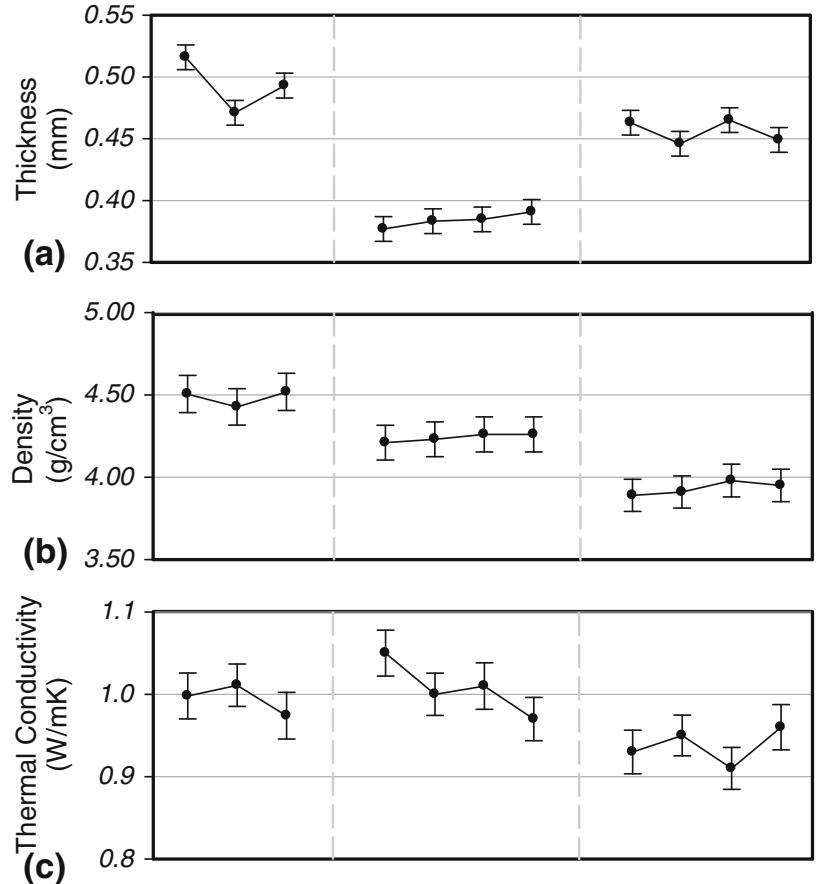

(c)
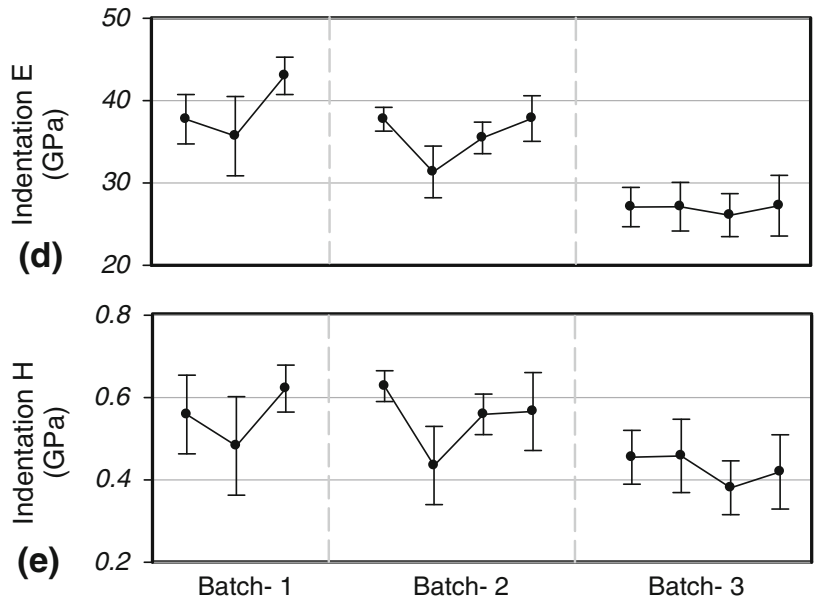

Fig. 10 Post spray deposition analysis and characterization for the three batches of YSZ coatings; batch 1 is data obtained from three separate coating runs, while batches 2 and 3 are single spray runs on a carousel set-up

Batch 2 displays the highest variability among its coatings, while variability within batch 1 is higher than that in batch 3 . These differences may be related to the level of porosity present in the coatings observed through microstructural characterization. Coatings from batch 2 are more porous than batches 1 and 3. Since, micro-indentation is the property characterization technique influenced by the local architecture of coating surrounding the indenter, higher porosity level is expected to give higher uncertainty in measurements, and thus variability.

\subsubsection{Assessing Coating Property Repeatability} Through Measurement of Nonlinear Properties. Postspray characterization using metallography, indentation, and thermal conductivity are prone to higher intrinsic

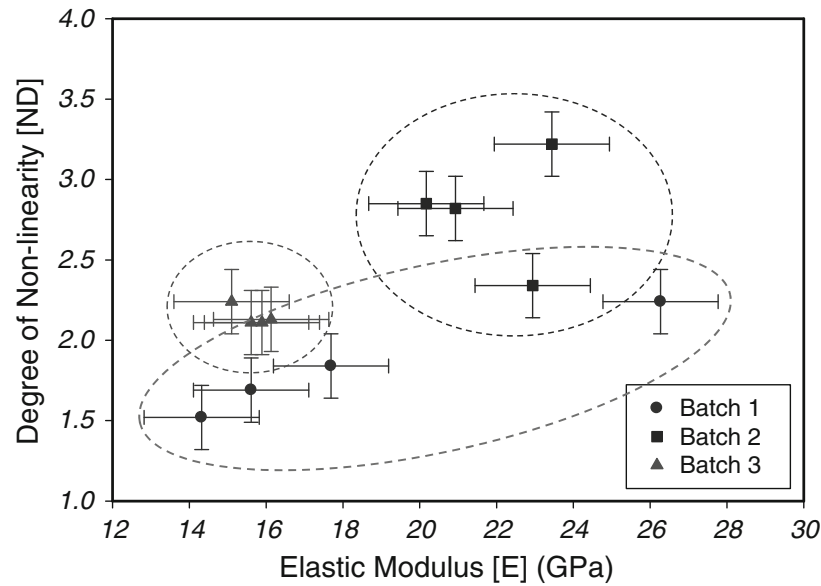

Fig. 11 Map of nonlinear elastic properties of plasma-sprayed YSZ coatings from the three batches reported in Fig. 5

variability associated not only with the technique itself, but also the need for destructive specimen preparation which has potential to introduce artifacts.

On the other hand, ECP sensor based on the principle of non-destructive in plane curvature measurement will minimize the source of errors associated with the multistep/destructive characterization techniques. Based on extensive development and testing of the ECP, it has been observed that the sensitivity of the nonlinear analysis is within an error of $3 \mathrm{GPa}$ and 0.3 for $E$ and ND, respectively.

Figure 11 provides the map of nonlinear elastic properties of all the samples from batches 1,2, and 3. Elastic modulus, the $x$-axis of the figure, is estimated from the linear region of the nonlinear stress-strain calculations, while degree of nonlinearity is calculated from the ratio of modulii between the linear portion of low- and high-strain regions (Ref 26, 28). Effectively, the elastic modulus captures the extent of defects in the system (e.g., pores and interfaces) while the nonlinearity to a first approximation describes the nature of the interaction among the material surfaces within these pores and interfaces. The error bars in the figure were calculated from multiple cycles of single specimen over several days. This quantification of nonlinear properties in terms of ND, thus, provides a robust platform for comparison.

Several important observations can be made from Fig. 11:

- All the coatings show a nonlinear elastic response consistent with our previous results (Ref 26). Different batches show different levels of variabilities in their nonlinear elastic properties. The resolution of nonlinear analysis technique provides sufficient fidelity for comparing subtle changes in process and coatings.

- There can be significant local variability in properties of sprayed YSZ coatings even when sprayed in a single run. These local variabilities can introduce global variability in properties. 
- Since, batches 2 and 3 were made on a rotating carousel, the coating formation mechanism is different from the case of batch 1 . They show higher nonlinearity presumably due to lower splat impact interval period. Variability among the coatings within the same batch, is maximum for batch 1 made over different days, and which includes start-stop of the plasma torch, different ambient conditions (humidity, temperature etc.), and many other factors that can increase the variability. Batch 3 was identical in all the aspects except for powder size distribution compared to batch 2. This results in different ND and $E$ values which notably also show a much smaller variability. From this observation, it can be concluded that a narrower powder size distribution has a more profound effect on the reproducibility of coatings in terms of their properties.

\subsection{Effect of Hardware Degradation in Particle States and Coatings: Assessment of the Reproducibility of the Process to Achieve Similar Coatings}

Further to investigation of process and coating repeatability through multiple runs, hardware changes (e.g., electrode degradation) can result in potentially significant changes to the process and many instances require modifications to reproduce a coating to meet the requisite specification. When hardware is changed, not only the specific torch parameters but also the location of the output responses in particle states can be affected. In order to examine these electrode degradation effects on process and coating reproducibility, three sets of process parameters (low, medium, and high in particle energy states-LE, ME, HE) were identified and particle states/ coating properties were monitored for each of these conditions using an "old" worn cathode and new cathode.
The old cathode was replaced by the new one after around $25 \mathrm{~h}$ of service having more than 50 start-stops of the plasma torch. The low, medium, and high conditions were selected from previous first-order process map runs. For each case, the particle state diagnostics were conducted following the procedures described in Sect. 2.3.1. AccuraSpray sensor was used for diagnostics. Further, the substrates preparation as well as the coatings deposition procedure was kept nominally identical (Sect. 2.3.2). Subsequent to the coating deposition, coating thicknesses and residual curvature were measured for comparison, and coatings were thermal cycled using the ECP to extract their nonlinear elastic properties.

Not surprisingly, a systemic shift in particles $T$ and $V$ were observed for the case of new cathode for a same set of process parameters (Fig. 12a). The temperatures for the three conditions were observed to be significantly higher, though the velocity changes were relatively close. These results are consistent with prior observations in the literature (Ref 15).

From the Fig. 13, it can clearly be observed that the new cathode increases the deposition efficiency (DE) associated with increase in particle temperature and resultant melt content. Furthermore, the improvement in DE in terms of the coating thickness increases by a different factor for each case: $4 \%$ for HE, $14 \% \mathrm{ME}$ and $11 \%$ for LE, suggesting the improvement is depending on the position in the process map. That is, the LE and ME conditions are more significantly affected by the electrode change as more particles are presumably at the cusp of melting.

Figure 12(b) presents the nonlinear elastic property map of the coatings from the two different batches. As an initial observation, it can be seen that the elastic modulus obtained from the curvature measurements is not significantly affected by nozzle change at least within the parametric framework considered here; however, the nonlinearity parameter is significantly affected. For both
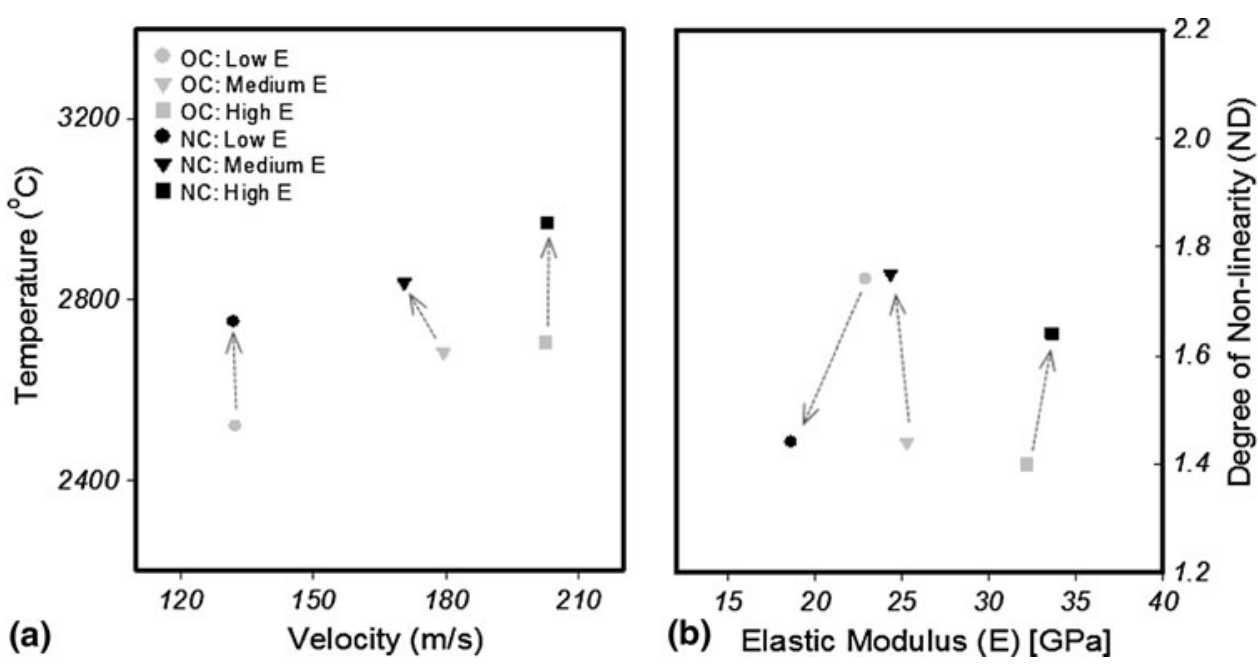

Fig. 12 Correlation of particles energies with their corresponding coatings' elastic properties. The figure also exhibits the shifts in (a) particle state parameters and (b) nonlinear elastic properties of the coatings due to cathode nozzle change (OC: Old Cathode, NC: New Cathode) 


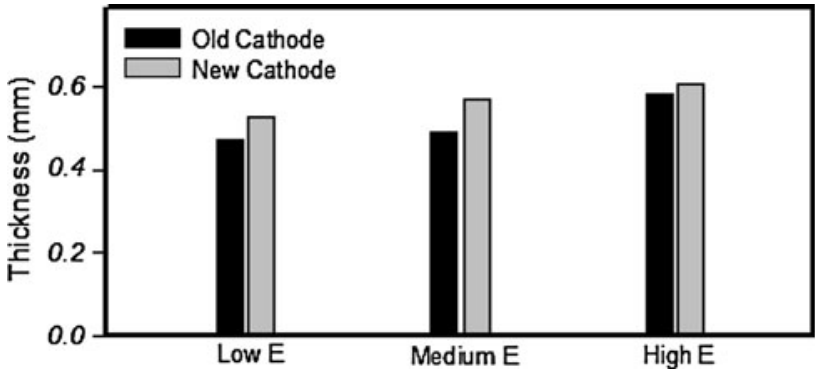

Fig. 13 Increase in thickness as a result of replacing a new cathode with a worn out old one

$\mathrm{ME}$ and $\mathrm{HE}$, the nonlinearity is somewhat higher perhaps related to enhanced particle flattening, while for the LE case, the nonlinearity shows a decrease. The exact mechanism of these changes requires further investigation through microstructural investigation, but it is worthwhile to point out that subtle changes in the process can affect the coating properties in complex ways.

\section{Using Non-Linear Properties to Compare Day-to-Day Variation in Production of Coatings}

\subsection{Part 1}

The above strategies can also be used to compare properties of commercial production coatings. In order to accomplish this, four sets of measurements were conducted:

(i) The first set comprised repeated measurements of nonlinear properties of a single coating produced at Stony Brook. This served as a benchmark to test intrinsic variability of the measurement apparatus and analysis procedure.

(ii) A second set of three coatings produced at Stony Brook through repeated spray runs, where due consideration to the entire process was made (e.g., particle state, substrate temperature etc.) to ensure the best possible reproducibility outcomes. Here, commercial powders and spray process parameters were utilized. This provides a framework for the best achievable reproducibility in a carefully controlled production setting.

(iii) Two sets of commercial production coatings were obtained from industrial collaborators. The two coating producers applied nominally the same procedure as those followed in a typical production coating protocol. No further details on the process conditions were available for these coatings, except that these coatings would nominally satisfy a standard procedure of meeting an engine coating specifications. The samples were sprayed onto aluminum substrates provided by Stony Brook to the industrial vendors.

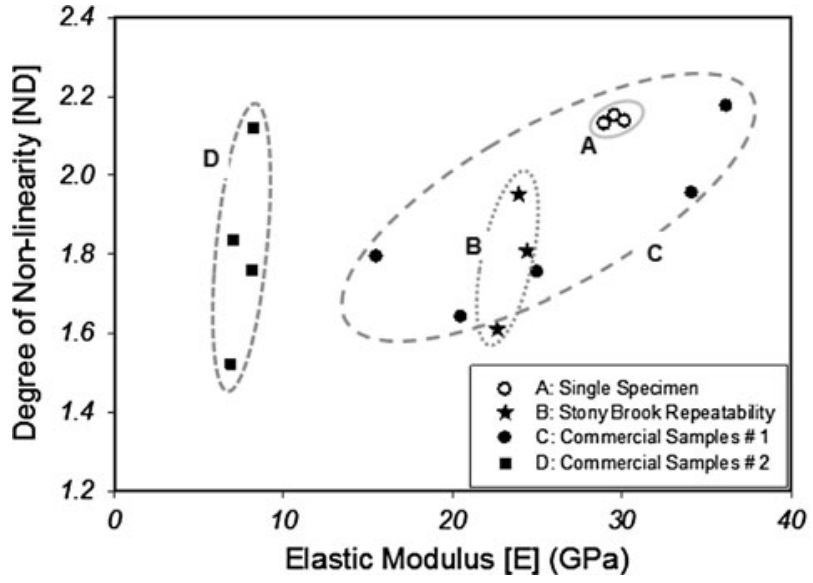

Fig. 14 Comparison on nonlinear properties of laboratory and production plasma-sprayed YSZ coatings. Repeated measurements on a single specimen display the intrinsic reliability of the technique

Since no other starting information was available on these industrial coatings, the analysis is restricted to ECP measurements and extraction of the nonlinear properties. All the coatings were subjected to low temperature thermal cycling studies at Stony Brook using identical procedures. Same ECP sensor set-up was used for curvature and temperature measurements, but as these samples were processed prior to furnace-based testing, the samples were cycled using a hand-held flame torch, and the cooling portion was used for the nonlinear analysis. The details of the earlier procedure is mentioned in a previous study (Ref 27). The nonlinear stress-strain behaviors of the coatings were investigated and the parameters of nonlinearity degree and elastic modulus were obtained. The results of this investigation are plotted in Fig. 14. The test itself is benchmarked through repeated measurement of the single specimen, which clearly illustrates that technique can yield highly reproducible results. When compared to this reference, the Stony Brook samples produced with due considerations of process and particle properties result in similar elastic modulii but somewhat different nonlinear behaviors (note that they were made at different times but within a span of few days). The two commercial batches of samples display different types of response. The commercial sample A shows significant variance in both modulus and nonlinearity, while the commercial sample B behaves similar to the Stony Brook repeatability samples, i.e., similar modulii but different nonlinearities.

The results present a rather dramatic picture of the day-to-day variability in coating properties which were hitherto indeterminable through traditional methods of thermal spray coating characterization. The results from the Stony Brook and Commercial B sample suggest that coatings with similar porosity (to a first approximation captured through the elastic modulus) can be prepared, but the nature of the interfaces can be subtly different. Environmental attributes such as humidity may a play a 
role here. This requires further investigation. It is clear that the proposed nonlinear elastic analysis is an effective as well as sensitive approach to assess coating-coating variability in plasma-sprayed YSZs.

\subsection{Part 2}

4.2.1 Integrated Assessment of Reliability in a Production Environment. The above results provide a framework to assess process and coating variability as applied to plasma-sprayed YSZ system. Plasma-sprayed YSZ represents perhaps the most complex of the thermal spray applications and as such it is envisioned that the progress derived there in can be extended to other thermal spray materials and processes. However, a true measure of the variability and methodologies to reduce them requires demonstration in an industrial setting. In order to accomplish this, collaborative experiments were conducted between Stony Brook and Tinker Air Force Base in a semi-production spray setting.

Three sets of experiments were carried out in a industrial production environment equipped with an F4 plasma spray system and three batches of coatings were deposited: TB1, TB2, and TB3. In the first instant (TB1), standard production parameters were utilized. Here, all the parameters including carrier gas flow was pre-set according to established procedure. In a second case (TB2), three additional experiments were conducted following the injection optimization procedure developed at Stony Brook. In this situation, the carrier gas flow was adjusted during each run, so as to achieve the same (optimized) plume position accessing the "sweet-spot" of the spray torch. For both TB1 and TB2, the particle states were monitored using AccuraSpray sensor and the coatings were deposited onto the ICP sensor. The ICP sensor allowed extraction of the evolving stress during deposition. The third batch (TB3) of 12 coatings was obtained over a range of a month from production spray booths. Standard production procedures employed routinely were applied during the deposition of ECP coupons. Subsequent to deposition, the coatings were brought back to Stony Brook for ex situ characterization of coating properties; including coating thickness and thermal cycling to extract nonlinear elastic properties.

Figure 15 displays the first-order particle $T-V$ map of the results obtained from AccuraSpray for TB1 and TB2. The results clearly demonstrate the advantage of injection optimization procedure. The experiments conducted with this procedure consistently showed an increase in both particle $T$ and $V$. Further, these conditions also resulted in smaller variability in particle states compared to those from standard condition where the carrier gas flow was chosen as a fixed parameter.

Table 6 provides the summary of the coating characteristics. Several important observations can be made from the results.

- Coatings produced with injection optimization resulted in consistently higher deposition efficiency as evidenced by the thickness per pass measurements.

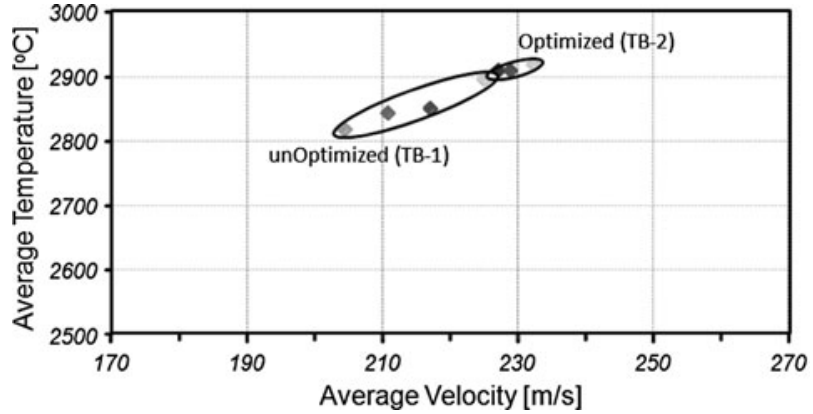

Fig. 15 Particle temperature and velocities for the two sets of spray runs described in Sect. 4.1

Table 6 Thickness per pass and in situ measured coating properties of two sets of plasma-sprayed TBC samples obtained in a production environment

\begin{tabular}{|c|c|c|c|c|}
\hline $\begin{array}{l}\text { Sample } \\
\text { details }\end{array}$ & $\begin{array}{c}\text { Thickness } \\
\text { per pass, } \mu \mathrm{m}\end{array}$ & $\begin{array}{c}\text { Evolving } \\
\text { stress, } \\
\text { MPa }\end{array}$ & $\begin{array}{c}\text { Thermal } \\
\text { stress, } \\
\text { MPa }\end{array}$ & $\begin{array}{c}\text { Residual } \\
\text { stress, MPa }\end{array}$ \\
\hline \multicolumn{5}{|c|}{ Without injection optimization } \\
\hline TB1-1 & 11.8 & 14.6 & -58.6 & -44 \\
\hline TB1-2 & 11.6 & 15.2 & -53.2 & -38 \\
\hline TB1-3 & 11.7 & 19.0 & -56.0 & -37 \\
\hline TB1-4 & 11.8 & 18.6 & -54.6 & -36 \\
\hline \multicolumn{5}{|c|}{ With injection optimization } \\
\hline TB2-1 & 12.2 & 13.1 & -52.7 & -39 \\
\hline TB2-2 & 12.4 & 13.7 & -53.7 & -40 \\
\hline TB2-3 & 12.3 & 14.9 & -54.9 & -40 \\
\hline
\end{tabular}

The TB-1 set follows standard production protocols while TB-2 utilizes and feedback control mechanism to optimize the injection location of the particles for each spray run

This can be directly related to the higher overall temperatures and velocities obtained during the spray runs. The variability in thickness was indistinguishable among the two sets of runs. This is not surprising given that the same powder was used.

- The nonlinearity and elastic modulus of the coatings produced using the injection optimization procedure (TB2) showed substantially lower variability compared to those produced via the standard condition (TB1). On the contrary, TB3 shows significantly large scatter on the ND- $E$ map.

Figure 16 captures this reduced variability in coating properties produced via the modified procedure as displayed nonlinearity-elastic modulus diagram. It is clear that the optimized coatings show significantly lower variability in the ND- $E$ space. In order to further illustrate the advantage of this procedure, Fig. 16 also incorporates production coatings which were obtained from various other spray booths within a facility and those produced with substantial gap between production runs. When compared in context of this larger set of data, the power of the proposed optimization procedure and the ability to monitor the variability through advanced measurement 


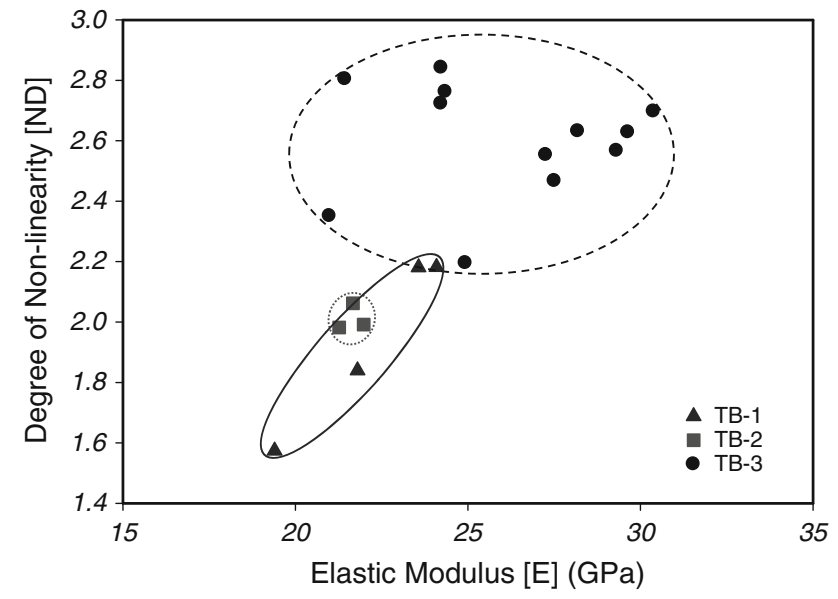

Fig. 16 Comparison of nonlinear properties of batches TB1, $\mathrm{TB} 2$, and TB3

and analysis concepts is demonstrated. Indeed, when combined with the thickness results (which is a measure of relative deposition efficiency among the various spray runs), the coatings produced using enhanced procedures are not only more reproducible but also makes the process more efficient, with concomitant benefits for both production and application.

\section{Summary and Conclusion}

This study presents integrated investigation of variabilities associated with the various sub-steps involved in plasma spray processing of thermal barrier coatings: from feedstock powder to coating properties. The advent of in situ process diagnostics as well as novel methods of coating characterization has, perhaps for the first time, revealed new insights into sources of variability in plasma spray. When these techniques are combined with carefully designed experimental strategies, they shed light into the intrinsic variabilities within the process as well as the measurements and provide a framework for process control.

The analysis reported within this article addresses "repeatability" of the process (i.e., variation associated with repeated identical experiments) and "reproducibility" of the process (describing both spatial and temporal variations). The former provides information on the intrinsic variabilities in each step of processing and measurement while the latter signifies achievable outcome. Together, these studies shed light into the overall reliability of the plasma spray process technology particularly for complex applications such as thermal barrier coatings.

The results from the integrated studies provide for the following conclusions:

- In general, the lot-to-lot variability in commercial feedstock distributions obtained from a single manufacturer is reproducible although small changes are observed when different manufacturers' feedstock are involved. Nevertheless, the results point to relatively high consistency in feedstock materials for plasma spray.

- Different particle state sensors report different absolute values of particle temperature and velocity, but when appropriate care in measurements is taken, they can provide reasonable information on average particle states.

- The variability in particle state is sensitive to parameter selection. The use of first-order maps allows examination of parameter specific variability allowing for process design to enhance reliability.

- When examined through careful experimentation, the particle states for plasma spray are surprisingly stable; however, the coatings can show both local and global variabilities which are only revealed through advanced property measurements.

- Tightening the feedstock size distribution allows for lower variability in design-relevant coating properties.

- Measurement of nonlinear elastic properties provides a novel means to assess coating variability.

- The results indicate that the scientific concepts as well as advanced measurement tools can be readily applied in a manufacturing environment with concomitant benefits to enhance process efficiency and coating reliability.

\section{Acknowledgments}

The authors gratefully acknowledge assistance provided by Jose Colmenares-Angulo, Dr. Brian Choi, Dr. Alfredo Valarezo, Dr. Yajie Liu, and Dr. Vasudevan Srinivasan. This research was supported in part by the NSF GOALI-FRG program under award CMMI 0605704 (co-funded by DMR), and by Air Force Research Laboratory Phase II SBIR program Thermal Barrier Coating Monitoring. The financial support through the industrial consortium for thermal spray technology is gratefully acknowledged.

\section{References}

1. V. Arnault, R. Mevrel, S. Alperine, and Y. Jaslier, Thermal Barrier Coatings for Aircraft Turbine Airfoils: Thermal Challenge and Materials, Rev. Metall. Cah. Inf. Tech., 1999, 96(5), p 585-597

2. P. Fauchais, Formation of Plasma-Sprayed Coatings, J. Therm. Spray Technol., 1995, 4(1), p 3-6

3. P. Fauchais, A. Grimaud, A. Vardelle, and M. Vardelle, Plasma Spraying: An Overview, Ann. Phys., 1989, 14(3), p 261-310 (in French)

4. P. Fauchais, Understanding Plasma Spraying, J. Phys. D: Appl. Phys., 2004, 37(9), p R86-R108

5. S. Sampath and R. McCune, Thermal-Spray Processing of Materials, MRS Bull., 2000, 25(7), p 12-14

6. S. Ben Naoua, M. El Ganaoui, H. Sammouda, and P. Fauchais, Rapid Solidefication Study of Stabilized Zirconia Under Plasma Spraying, High Temp. Mater. Processes, 2006, 10(2), p 185-196 
7. S. Sampath and H. Herman, Rapid Solidification and Microstructure Development During Plasma Spray Deposition, J. Therm. Spray Technol., 1996, 5(4), p 445-456

8. P. Bengtsson and T. Johannesson, Characterization of Microstructural Defects in Plasma-Sprayed Thermal Barrier Coatings, J. Therm. Spray Technol., 1995, 4(3), p 245-251

9. S. Alperine and L. Lelait, Microstructural Investigations of Plasma-Sprayed Yttria Partially-Stabilized Zirconia Tbc (in Relation to Thermomechanical Resistance and High-Temperature Oxidation Mechanisms), J. Eng. Gas Turbines Power, 1994, 116(1), p 258-265

10. A.J. Allen, G.G. Long, H. Boukari, J. Ilavskya, A. Kulkarni, S. Sampath, H. Herman, and A.N. Goland, Microstructural Characterization Studies to Relate the Properties of Thermal-Spray Coatings to Feedstock and Spray Conditions, Surf. Coat. Technol., 2001, 146, p 544-552

11. D.R. Clarke and C.G. Levi, Materials Design for the Next Generation Thermal Barrier Coatings, Annu. Rev. Mater. Res. 2003, 33, p 383-417

12. C. Moreau, J.F. Bisson, R.S. Lima, and B.R. Marple, Diagnostics for Advanced Materials Processing by Plasma Spraying, Pure Appl. Chem., 2005, 77(2), p 443-462

13. J.R. Fincke, D.C. Haggard, and W.D. Swank, Particle Temperature Measurement in the Thermal Spray Process, J. Therm. Spray Technol., 2001, 10(2), p 255-266

14. P.F.M. Vardelle, Plasma Spray Processes: Diagnostics and Control?, Pure Appl. Chem., 1994, 5(3), p 205-212

15. C. Moreau and L. Leblanc, Optimization and Process Control for High Performance Thermal Spray Coatings, Durable Surf., 2001, 197, p 27-57

16. V. Srinivasan, M. Friis, A. Vaidya, T. Streibl, and S. Sampath, Particle Injection in Direct Current Air Plasma Spray: Salient Observations and Optimization Strategies, Plasma Chem. Plasma Process., 2007, 27(5), p 609-623

17. V. Srinivasan, A. Vaidya, T. Streibl, M. Friis, and S. Sampath, On the Reproducibility of Air Plasma Spray Process and Control of Particle State, J. Therm. Spray Technol., 2006, 15(4), p 739-743

18. S. Sampath, V. Srinivasan, A. Valarezo, A. Vaidya, and T. Streibl, Sensing, Control, and In Situ Measurement of Coating Properties: An Integrated Approach Toward Establishing Process-Property Correlations, J. Therm. Spray Technol., 2009, 18(2), p 243-255

19. V. Srinivasan, "A Critical Assessment of In-Flight Particle State During Plasma Spraying of YSZ and its Implications on Coating Properties and Process Reliability," Ph.D. Thesis, Stony Brook University, 2007

20. A. Vaidya, V. Sirinivasan, T. Streibl, M. Friis, W. Chi, and S. Sampath, Process Maps for Plasma Spraying of Yttria-Stabilized Zirconia: An Integrated Approach to Design, Optimization and Reliability, Mater. Sci. Eng. A, 2008, 497(1-2), p 239-253

21. A.A. Kulkarni, A. Goland, H. Herman, A.J. Allen, J. Ilavsky, G.G. Long, and F. De Carlo, Advanced Microstructural Characterization of Plasma-Sprayed Zirconia Coatings Over Extended Length Scales, J. Therm. Spray Technol., 2005, 14(2), p 239-250

22. H. Wang and R.B. Dinwiddie, Characterization of Thermal Barrier Coatings Using Thermal Methods, Adv. Eng. Mater., 2001, 3(7), p 465-468

23. F. Tang and J.M. Schoenung, Evolution of Young's Modulus of Air Plasma Sprayed Yttria-Stabilized Zirconia in Thermally Cycled Thermal Barrier Coatings, Scripta Mater., 2006, 54(9), p 1587-1592
24. A. Kucuk, C.C. Berndt, U. Senturk, R.S. Lima, and C.R.C. Lima, Influence of Plasma Spray Parameters on Mechanical Properties of Yttria Stabilized Zirconia Coatings. I: Four Point Bend Test, Mater. Sci. Eng. A, 2000, 284(1-2), p 29-40

25. A. Kucuk, C.C. Berndt, U. Senturk, and R.S. Lima, Influence of Plasma Spray Parameters on Mechanical Properties of Yttria Stabilized Zirconia Coatings. II: Acoustic Emission Response, Mater. Sci. Eng. A, 2000, 284(1-2), p 41-50

26. T. Nakamura and Y.J. Liu, Determination of Nonlinear Properties of Thermal Sprayed Ceramic Coatings Via Inverse Analysis, Int. J. Solids Struct., 2007, 44(6), p 1990-2009

27. Y. Liu, T. Nakamura, V. Srinivasan, A. Vaidya, A. Gouldstone, and S. Sampath, Non-Linear Elastic Properties of PlasmaSprayed Zirconia Coatings and Associated Relationships with Processing Conditions, Acta Mater., 2007, 55(14), p 4667-4678

28. Y.J. Liu, T. Nakamura, G. Dwivedi, A. Valarezo, and S. Sampath, Anelastic Behavior of Plasma-Sprayed Zirconia Coatings, J. Am. Ceram. Soc., 2008, 91(12), p 4036-4043

29. R.E. Taylor, Thermal Conductivity Determinations of Thermal Barrier Coatings, Mater. Sci. Eng. A, 1998, 245(2), p 160-167

30. W. Chi, "Thermal Transport Properties of Thermally Sprayed Coatings: An Integrated Study of Materials, Processing and Microstructural Effects," Ph.D. thesis, Stony Brook University, 2007

31. A. Vaidya, "Process Maps for Thermal Spray: A Fundamental Approach to Process-Property Relationships," Ph.D. thesis, Stony Brook University, 2008

32. S. Sampath, X. Jiang, A. Kulkarni, J. Matejicek, D.L. Gilmore, and R.A. Neiser, Development of Process Maps for Plasma Spray: Case Study for Molybdenum, Mater. Sci. Eng. A, 2003, 348(1-2), p 54-66

33. W. Zhang, L.L. Zheng, H. Zhang, and S. Sampath, Study of Injection Angle and Carrier Gas Flow Rate Effects on Particles In-Flight Characteristics in Plasma Spray Process: Modeling and Experiments, Plasma Chem. Plasma Process., 2007, 27(6), p 701716

34. A. Vaidya, S. Sampath, and H. Herman, Influence of Process Variables on the Plasma Sprayed Coatings: An Integrated Study, International Thermal Spray Conference (ITSC), 2001 (Singapore) (Materials Park, OH), ASM International, 2001

35. J. Matejicek and S. Sampath, In Situ Measurement of Residual Stresses and Elastic Moduli in Thermal Sprayed Coatings. Part 1: Apparatus and Analysis, Acta Mater., 2003, 51(3), p 863-872

36. J. Matejicek, S. Sampath, D. Gilmore, and R. Neiser, In Situ Measurement of Residual Stresses and Elastic Moduli in Thermal Sprayed Coatings. Part 2: Processing Effects on Properties of Mo Coatings, Acta Mater., 2003, 51(3), p 873-885

37. T. Streibl, A. Vaidya, M. Friis, V. Srinivasan, and S. Sampath, A Critical Assessment of Particle Temperature Distributions During Plasma Spraying: Experimental Results for YSZ, Plasma Chem. Plasma Process., 2006, 26(1), p 73-102

38. T. Wentz, "Thermal Spray Process Variability and Methods of Control," MS thesis, Stony Brook University, 2009

39. Y.P. Wan, H. Zhang, X.Y. Jiang, S. Sampath, and V. Prasad, Role of Solidification, Substrate Temperature and Reynolds Number on Droplet Spreading in Thermal Spray Deposition: Measurements and Modeling, J. Heat Transfer, 2001, 123(2), p 382-389

40. W. Zhang and S. Sampath, A Universal Method for Representation of In-Flight Particle Characteristics in Thermal Spray Processes, J. Therm. Spray Technol., 2009, 18(1), p 23-34

41. W.G. Chi, S. Sampath, and H. Wang, Microstructure-Thermal Conductivity Relationships for Plasma-Sprayed Yttria-Stabilized Zirconia Coatings, J. Am. Ceram. Soc., 2008, 91(8), p 2636-2645 